\title{
Les tatouages d'Ötzi et la petite chirurgie traditionnelle
}

\section{Ötzi’s Tattoos and Traditional Minor Surgery}

\author{
Luc Renaut $^{1}$ \\ 88, rue Bobillot, 75013 Paris, France \\ Disponible sur Internet le 10 avril 2004
}

\section{Résumé}

Cet article reprend le dossier des tatouages portés par Ötzi, cet homme du Néolithique final retrouvé en 1991 dans un glacier des Alpes italo-autrichiennes. Les équipes autrichiennes et italiennes ont déjà avancé un certain nombre d'hypothèses dont la principale, qui consiste à reconnaître une fonction thérapeutique aux tatouages d'Ötzi, n'est pas remise en cause. D'autres toutefois peuvent être rapidement infirmées - ainsi la soi-disante « cautérisation tibétaine » de L. Capasso - ou bien méritent une discussion plus approfondie, comme la tentative autrichienne d'établir un lien entre la localisation des tatouages d'Ötzi et celle des points d'acupuncture. Ces hypothèses contestables sont imputables à une méconnaissance des anciennes thérapeutiques fondées sur des interventions de petite chirurgie. Il a donc paru nécessaire de réunir ici les données ethnographiques et historiques disponibles sur la puncture, le tatouage et la cautérisation à destination thérapeutique. La réunion de ces matériaux pourtant postérieurs à Ötzi constitue le préalable indispensable à toute hypothèse sur les conceptions et les pratiques thérapeutiques protohistoriques.

(C) 2004 Elsevier SAS. Tous droits réservés.

\begin{abstract}
This paper takes a new look at the case of the tattoos worn by Ötzi, a late Neolithic man discovered in 1991 in a small glacier of the Southeast Alps, near the Italo-Austrian border. The Austrian and Italian teams have already advanced several hypotheses. The main one, which suggests that Ötzi was tattooed for therapeutic purposes, is not contested. Others, however, can be briefly invalidated (the "tibetan cauterization" alleged by L. Capasso), while still others call for more in-depth discussion (the Austrian team's attempt to establish a link between the location of Ötzi's tattoos and the topography of acupuncture-points). Questionable hypotheses as these result from a lack of knowledge about the ancient treatments using minor surgery. The aim of this paper is thus to assemble available ethnogra-
\end{abstract}

Adressee-mail : luc.renaut@laposte.net (L. Renaut). 
phical and historical data about therapeutic puncturing, tattooing and cauterization. Even though such material postdates Ötzi's era, it constitutes an essential prerequisite to the formulation of any hypothesis about protohistorical therapeutic concepts and practices.

(C) 2004 Elsevier SAS. Tous droits réservés.

Mots clés : Acupuncture ; Homme des glaces ; Moxibustion ; Cautérisation ; Paléopathologie ; Tatouage

Keywords: Acupuncture; Ice man; Moxibustion; Cauterisation; Paleopathology; Tattooing

\section{Introduction}

Les plus anciens tatouages connus à ce jour ont été livrés de la manière la plus directe qui soit, par celui-là même qui les a portés, et dont le corps étonnamment bien conservé a été découvert il y a un peu plus d'une dizaine d'années dans les Alpes italo-autrichiennes. Plusieurs membres du Laboratoire d'Anthropologie de Chieti (Italie), emmenés par Luigi Capasso, ont donné ici même une synthèse complète de cette découverte ${ }^{2}$. Notre présentation sera donc succincte.

Le 19 septembre 1991, suite à un été particulièrement chaud qui favorisa la fonte de sa masse neigeuse, un petit glacier $(3200 \mathrm{~m})$ de la chaîne de l'Ötztal ou du Similaun (où l'Hauslabjoch culmine à $3278 \mathrm{~m}$ ), niché au sommet de la vallée de Senales, livra à un couple de randonneurs, Érika et Helmut Simon, un corps inerte bientôt promis à la célébrité internationale. Avant qu'on eût établi que la découverte avait été faite sur le sol italien (à moins de 100 mètres de la frontière) $)^{3}$, des médecins légistes autrichiens vinrent dégager celui qu'ils surnommèrent bientôt « Ötzi », et l'emmenèrent reposer à l'Institut médicolégal de l'Université d'Innsbruck, où il demeura pendant six ans à la disposition des équipes coordonnées par l'archéologue Konrad Spindler ${ }^{4}$. En janvier 1998, Ötzi retourna en Italie 5 , à Bolzano, dans un musée où fut aménagé pour lui un caisson d'exposition reproduisant les conditions du glacier d'origine $\left(-6,5^{\circ} \mathrm{C}\right.$ et $98 \%$ d'humidité).

En février 1992, les laboratoires d'Oxford et de Zurich datèrent le corps au C14 d'environ 5300 ans BP (c. 3300 avant notre ère). Ses caractéristiques anthropologiques et son patrimoine génétique montrent par ailleurs qu'Ötzi est apparenté avec les habitants actuels d'Europe centrale et septentrionale ${ }^{6}$. Ötzi n'a pas été enseveli intentionnellement à l'altitude où il a été découvert. La mort l'a surpris en solitaire, et les vestiges archéologiques qui l'accompagnaient ne constituent pas un dépôt funéraire, mais l'équipement d'un

\footnotetext{
${ }^{1}$ Doctorant à l'École Pratique des Hautes Études (Paris Sorbonne), $5^{\mathrm{e}}$ section (Sciences religieuses). Soutenance de thèse prévue en 2004 : La marque corporelle dans l'Antiquité et le problème de la signation religieuse (dir. Alain le Boulluec).

${ }^{2}$ Capasso et al., 1999.

${ }^{3}$ Le fait fut constaté officiellement au début du mois d'octobre 1991. Dans son édition du 26 septembre 1991 (p. 16), le quotidien Le Monde localise encore la découverte en Autriche.

${ }^{4}$ Les scientifiques de l'Université d'Innsbruck ont depuis lors publié trois volumes collectifs sur le sujet : Der Mann im Eis, vol. 1, 1992 ; Der Mann im Eis, vol. 2, 1995 et enfin The Man in the Ice, vol. 3, 1996.

${ }^{5}$ Le Monde, 17 janvier 1998, p. 24, et Libération, 27 janvier 1998, p. 30.

${ }^{6}$ Sjøvold, 1992, p. 196; Stone, 2000, p. 2254.

${ }^{7}$ Des examens (radiographies et scanner) menés en juillet 2001 ont montré la présence d'une pointe de flèche en silex dans l'épaule gauche qui pourrait avoir sectionné l'artère du bras gauche et avoir dès lors entraîné un décès
} 
chasseur en expédition : des restes de vêtement ${ }^{8}$, une hache en cuivre à manche coudé ${ }^{9}$, un poignard en silex avec sa gaine, un arc, un carquois, 14 flèches ${ }^{10}$, un retouchoir en bois de tilleul avec pointe en silex, les restes d'une hotte et ceux d'un petit récipient en écorce de bouleau servant à transporter des braises. Enfin, Ötzi transportait un sac-ceinture en cuir rempli d'une masse de Fomes fomentarius (un champignon utilisé comme amadou depuis l'Antiquité) et contenant trois outils en silex et une alène d'os ${ }^{11}$. Sur le site du Similaun ont aussi été retrouvés deux morceaux de Piptoporus betulinus (Polypore spécifique du bouleau) perforés et passés sur un lacet de cuir ${ }^{12}$.

Dès le 20 septembre 1991, des traits sombres avaient été repérés sur le dos d'Ötzi. D'autres tatouages furent découverts au cours de l'année 1992. La plupart se présentaient sous la forme de groupes de traits verticaux parallèles répartis sur les lombes et les jambes. On remarqua aussi deux petites croix, l'une à l'intérieur du genou droit, l'autre à proximité de la malléole externe de la cheville gauche. Les premiers rapports et les premières analyses furent publiés en 1992 et 1993 par L. Capasso et K. Spindler ${ }^{13}$; mais tous deux, trompés par les photographies, exagérèrent parfois les dimensions des tatouages. Il fallut attendre 1995 pour que paraisse un inventaire détaillé et réaliste des tatouages d'Ötzi, dressé sous les auspices du médecin suédois Torstein Sjøvold ${ }^{14}$. Ce rapport révéla en outre l'existence de deux tatouages supplémentaires sur le mollet droit, mis en évidence grâce à des clichés infrarouges pris le 13 août $1993^{15}$. La restitution d'ensemble que nous proposons (Fig. 1) se fonde sur les dimensions relevées par l'équipe du professeur Sjøvold ${ }^{16}$. Les particules de pigment (carbonées ?) retrouvées sur certains tatouages d'Ötzi au niveau du tissu conjonctif sont en conformité avec les procédés traditionnels du tatouage ${ }^{17}$ : lésion du tégument, puis pommadage d'une matière colorante à base de suie ou de cendre délayée. Il est regrettable que des analyses histologiques n'aient pas été conduites au niveau des deux lignes

rapide par hémorragie (l'une des radiographies est reproduite dans Arnaud, 2002 : p. 98). Ce diagnostic est corroboré par le fait que la plaie n'est pas cicatrisée : la pointe de silex n'est donc pas le vestige d'un accident ancien dont Ötzi se serait remis, mais la cause directe de sa mort (examens coordonnés par E. Egarter, cf. Quilici, 2002).

${ }^{8}$ Fragments d'un manteau, d'un pantalon et d'un pagne, tous en peau de chèvre ; restes de chaussures (en cuir avec rembourrage de foin) ; petit sac-ceinture ; toque en fourrure d'ours (Capasso et al., 1999 : p. 457).

${ }^{9}$ L'âge d'Ötzi correspond à celui du Cuivre I de l'Italie septentrionale (c. 3400/3300 - 2900/2800 av. J.-C.). Cf. les remarques du palethnologue milanais : Marinis, 1997, p. 52.

${ }^{10}$ À pointes de silex (des pointes en bois de cerf ont aussi été retrouvées dans le carquois).

${ }^{11}$ Spindler, 1993 (1994), p. 108-113 et photographie en ex folio, p. 178-179.

${ }^{12}$ Cf. discussion infra, p. 29 sq.

${ }^{13}$ Capasso et al., 1992 ; Capasso, 1993 ; Spindler, 1993 (1994), p. 167-169.

${ }^{14}$ Sjøvold et al., 1995.

${ }^{15}$ Ibid., Figs. 4-5 et p. 283. Le rayonnement infrarouge pénètre la peau plus profondément que la lumière normale et permet de révéler des colorations rendues indistinctes par le dessèchement de la surface de la peau.

${ }^{16}$ Ibid., p. 285 (tableau synthétique).

${ }^{17}$ Des biopsies ont été prélevées sur un trait de chacun des groupes a, j, et m (cf. Fig. 1) par une équipe néerlandaise dirigée par le dermatologue Eddy Van der Velden (Van der Velden et al., 1995). Après analyse macroscopique, l'équipe s'avoue toutefois incapable de dire «si les particules de pigments sont de la suie ou une autre substance » et parle de puncture sans se demander si les tatouages n'auraient pas pu être obtenus par scarification (ibid., p. 276). L'état du tissu conjonctif d'Ötzi permet-il d'apporter des éléments de réponse sur ce point? Il faudrait poser la question aux scientifiques néerlandais. 


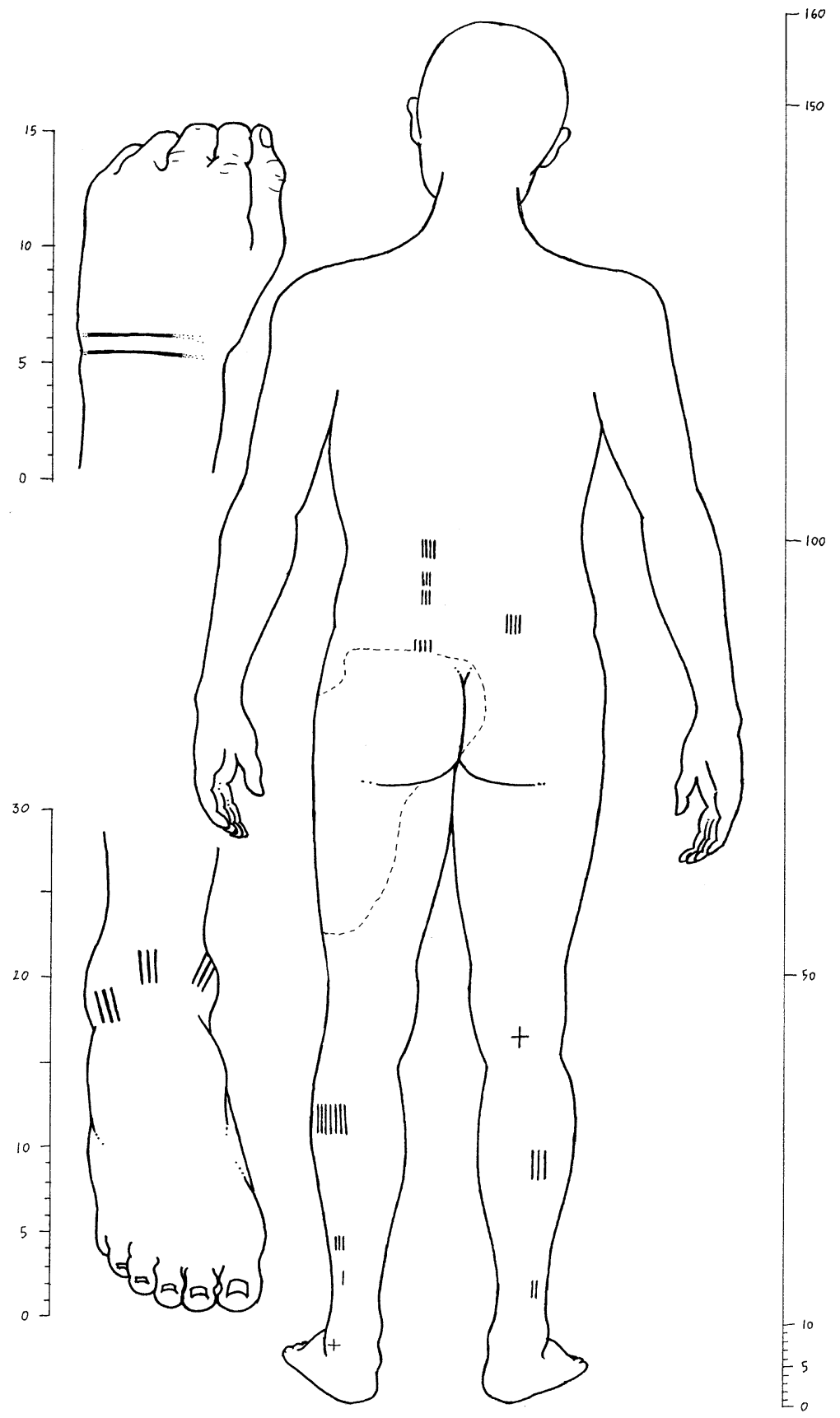

Fig. 1. Restitution des tatouages d'Ötzi (Luc Renaut).

Fig. 1. Restoration of Ötzi's tattoos (Luc Renaut). 
parallèles qu'Ötzi exhibe sur le plat supérieur du poignet gauche ${ }^{18}$; en effet, leur hétérogénéité faisant douter qu'il s'agisse de véritables tatouages ${ }^{19}$, seule l'absence constatée des particules de pigment aurait permis de confirmer ce soupçon.

\section{2. État de la question}

\subsection{Diagnostic arthrosique et musculaire}

Konrad Spindler, Luigi Capasso et d'autres chercheurs ont à juste titre dénié aux tatouages d'Ötzi toute fonction décorative, en privilégiant l'hypothèse thérapeutique. Deux arguments vont dans ce sens : la localisation des tatouages sur des zones habituellement recouvertes par le vêtement et l'aspect basique et répétitif des motifs, qui semble exclure une fonction décorative et/ou figurative. Restait à déterminer l'affection pour laquelle Ötzi aura jugé bon de recourir au tatouage. Pour répondre à cette question, les chercheurs autrichiens se sont fondés sur l'interprétation des radiographies et des scanographies du squelette d'Ötzi proposée par Dieter zur Nedden et Klaus Wicke ${ }^{20}$ :

«La radiographie de la colonne vertébrale montre que les vertèbres sont de dimension et de forme normales. Les intervalles des disques intervertébraux sont particulièrement minces. Cela dénote des phénomènes isolés de réduction (Vacuumphänomene), et au niveau lombaire, nous avons pu constater des altérations dégénératives légères à moyennes (diskrete bis mittelgradige degenerative Veränderungen) indiquant une ostéochondrose ainsi qu'une légère spondylite (im Sinne eine Osteochondrose und auch einer leichten Spondylose). [...] De même, Les articulations du genou, et surtout les articulations tibiotarsiennes, montrent des signes d'usure à moyen degré (Abnützungserscheinungen mäßigen Grades) ». ${ }^{21}$ "L'image au scanner montre aussi les altérations dégénératives des vertèbres lombaires mentionnées plus haut ${ }^{22}$.

Les Autrichiens remarquèrent que les lésions arthrosiques moyennes d'Ötzi affectaient des zones où des tatouages avaient été pratiqués : lombaires (où se concentrent cinq groupes de tatouages), genoux (croix à l'intérieur du genou droit) et chevilles (croix sur la malléole externe de la cheville gauche, et trois groupes tatoués autour de la cheville droite). Puisque les cinq groupes de traits tatoués sur les mollets ne pouvaient évidemment pas correspondre à une douleur articulaire, Autrichiens et Italiens ont suggéré qu'on ait cherché à traiter là

\footnotetext{
${ }^{18}$ Regrets exprimés par Sjøvold et al., 1995, p. 284.

${ }^{19}$ Luigi Capasso imagine que ces marques ont pu être provoquées post-mortem par des lanières serrées autour de la manche du vêtement (Capasso, 1993 : p. 178-179). Torstein Sjøvold et ses collègues pensent eux aussi «à des lanières composées de cuir ou de peau », comme celles sur lesquelles sont enfilés les champignons séchés retrouvés aux côtés d'Ötzi, ou bien à des lanières qui pourraient avoir servi « à la fixation d'une plaque de protection sur le bras, comme celles qu'on emploie pour le tir à l'arc » (Sjøvold et al., 1995 : p. 284).

${ }^{20}$ Diagnostic repris par Spindler, 1993 (1994), p. 172 et par Capasso et al., 1999, p. 461.

21 Zur Nedden et Wicke, 1992, p. 133-134. Les auteurs ne reproduisent ni radiographie ni scanner des lombaires et des genoux. Une radiographie (peu lisible) de la cheville droite se trouve toutefois reproduite p. 135, Fig. 4.

22 Ibid., p. 142.
} 


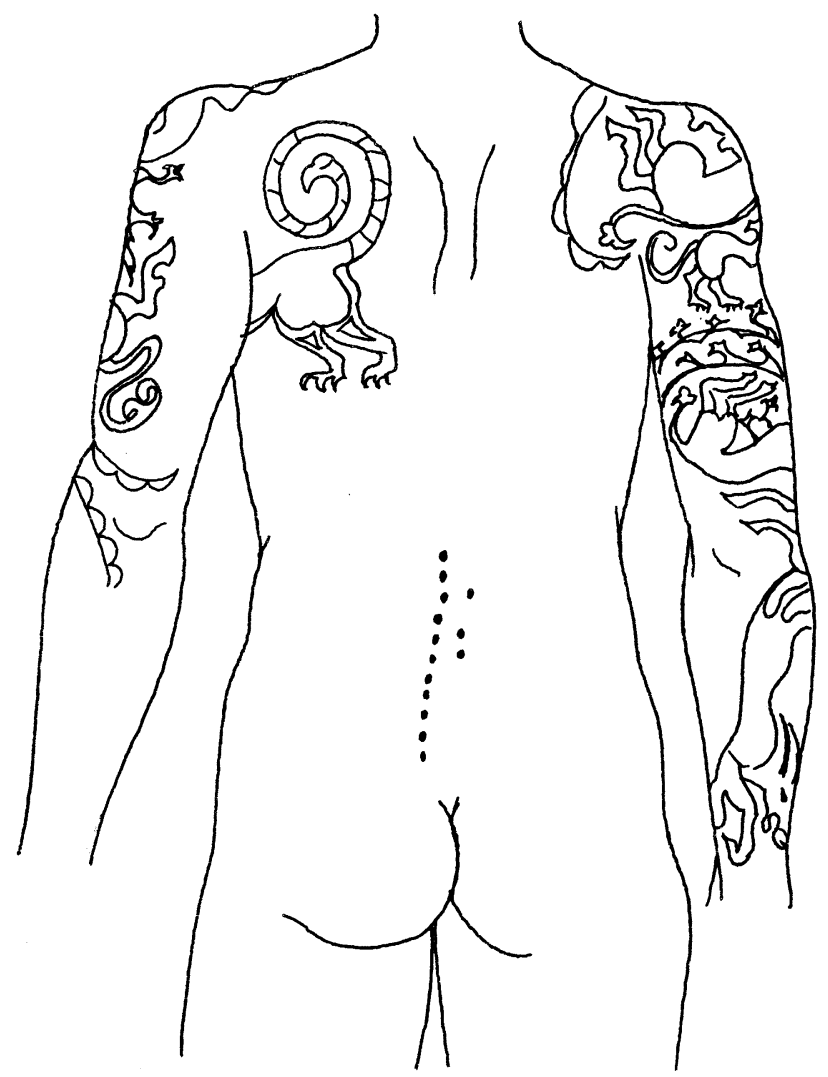

Fig. 2. L'homme de Pazyryk. Calque d'après Rudenko, 1949b, p. 135, Fig. 1 b.

Fig. 2. The Pazyryk man. Drawing after Rudenko, 1949b, p. 135, Fig. 1b.

une affection de type musculaire (courbature, contracture ou déchirure) ${ }^{23}$. Une fois ce diagnostic établi, les deux équipes ont recherché des parallèles historiques.

\subsection{Vers les steppes d'Asie centrale et les plateaux du Tibet}

Un premier rapprochement s'imposa de lui-même avec une autre momie tatouée découverte en 1948 dans l'Altaï sibérien (kourgane de Pazyryk, III ${ }^{\mathrm{e}}$ siècle av. J.-C.). Bien que cet «Homme de Pazyryk» soit principalement connu pour ses riches tatouages zoomorphes ${ }^{24}$, il présente également, au dos et à la cheville droite, des tatouages punctiformes : une ligne verticale de onze points, à gauche des vertèbres lombaires, jouxte une ligne similaire de trois points placée à droite (Fig. 2), tandis qu'une ligne incurvée de six points a été tatouée

\footnotetext{
${ }^{23}$ Spindler, 1993 (1994), p. 173 ; Capasso, 1993, p. 181 ; Capasso et al., 1999, p. 463.

${ }^{24} \mathrm{D}$ 'autres tatouages de ce type ont été retrouvés depuis dans la même région sur deux autres momies (l'une féminine, l'autre masculine, cf. Polosmak, 2000).
} 
à l'extérieur de la cheville droite ${ }^{25}$. La correspondance topographique de ces tatouages avec ceux d'Ötzi justifie pleinement le parallèle : même si l'on doit déplorer avec K. Splindler qu'aucune radiographie du squelette n'ait été prise à l'époque de la découverte ${ }^{26}$, on peut raisonnablement supposer que les tatouages punctiformes de l'Homme de Pazyryk répondaient eux aussi à des préoccupations thérapeutiques. Autrichiens et Italiens reprirent les réflexions de Sergei Ivanovitch Rudenko, l'inventeur de l'Homme de Pazyryk, qui avait le premier attiré l'attention sur les cautérisations thérapeutiques attribuées par la Collection hippocratique aux Nomades scythes ${ }^{27}:$ «Tu trouveras beaucoup de Scythes - dont tous ceux qui sont nomades - cautérisés (kekaumenous) aux épaules, aux bras, aux poignets, à la poitrine et aux lombes ${ }^{28}$. Selon l'auteur grec, qui cherche une explication conforme à sa propre théorie des contraires ${ }^{29}$, ce traitement vient corriger la nature humide et molle des Scythes due au climat de leurs contrées (i.e. l'Ukraine actuelle). La cautérisation, en asséchant les articulations, est censée remédier chez eux à une espèce de rhumatisme chronique et faciliter ainsi les activités de torsion et de détente qu'exige le lancer de javelot. À l'occasion de la découverte d'Ötzi, Renate Rolle ${ }^{30}$ publia une synthèse des travaux relatifs à l'Homme de Pazyryk dans laquelle il établit un rapprochement entre les anciennes cautérisations scythes et celles que la médecine populaire tibétaine utilise encore aujourd'hui, en donnant sur ces dernières les précisions suivantes :

«On se sert de différents outils, depuis le foyer brûlant d'une pipe (observé au milieu du XIX ${ }^{e}$ siècle) jusqu'à divers instruments cautérisants comme le poinçon ou le fer rouge. Encore aujourd'hui, la médecine populaire tibétaine brûle des herbes (Kräuter) sur la peau pour soigner le rhumatisme, l'infécondité ou le mal de dos ${ }^{31}$. Garnie (gefüllt) d'herbes enroulées (gewickelten Kräutern), la pointe (Spitze) d'un fer rouge ardent est posée sur la peau. » ${ }^{32}$

Pour tout le passage que nous avons cité, Renate Rolle ne donne aucune référence bibliographique ; nous nous sommes de notre côté entretenu personnellement avec Fernand Meyer, qui a publié plusieurs photographies illustrant les différents procédés de cautérisation employés aujourd'hui au Tibet ${ }^{33}$. Il faut en effet donner quelques éclaircissements sur la technique décrite en dernier lieu par Renate Rolle, car elle a conduit L. Capasso à des hypothèses fantaisistes. Les médecins tibétains utilisent un petit ressort hélicoïdal (fil de

\footnotetext{
${ }^{25}$ Dans son article de 1949 (Rudenko, 1949b : p. 141), S.I. Rudenko qualifie ces tatouages punctiformes de «petites boules » ou «billes », (шарики) puis de «petits cercles » (кружки diminutif de круг, « cercle »), et joint un dessin (p. 135, Fig. 1b) ou les tatouages dorsaux sont figurés par des petits cercles évidés (noircis sur notre Fig. 2). Mais il s'agit là sans doute d'une convention, abandonnée par la suite : la figure reproduite dans la traduction anglaise de 1970 donne en effet aux tatouages lombaires l'aspect de petits disques ou de gros points (Rudenko, 1970 : p. 109, Fig. 52).

${ }^{26}$ Spindler, 1993 (1994), p. 172. Le squelette de l'Homme de Pazyryk semble avoir disparu depuis.

${ }^{27}$ Rudenko, 1970, p. 113.

${ }^{28}$ Airs, eaux, lieux, 20, 1.

${ }^{29}$ Dans la médecine grecque, on soigne le froid par le chaud, l'humide par le sec, et vice-versa.

${ }^{30}$ Institut de Préhistoire, Université de Hambourg.

${ }^{31}$ Il s'agit là du procédé bien connu de la moxibustion, cf. infra, n. 51.

32 Rolle, 1992, p. 355.

${ }^{33}$ Meyer, 1988, p. $188-197$; Figs. 46-50.
} 
cuivre ou d'or enroulé sur lui-même) dont l'extrémité inférieure, rectiligne, forme la pointe qui sera appliquée sur la peau. Dans les spirales du ressort, on laisse se consumer un petit rouleau de feuilles de Gerbera séchées. La chaleur ainsi produite est transmise par la pointe de l'instrument jusqu'à la peau. Cette cautérisation indirecte, dite de l'aiguille d'or (gser-khab), est appliquée au sommet du crâne et ne laisse aucune cicatrice ${ }^{34}$.

Dans une contribution de 1993 consacrée aux tatouages d'Ötzi, Luigi Capasso livrait une première paraphrase plutôt correcte de la notice de Renate Rolle ${ }^{35}$, puis, après avoir évoqué les cautérisations scythes, supposait que les tatouages d'Ötzi devaient être le résultat d'une cautérisation indiquée pour le traitement de l'arthrose ${ }^{36}$. Mais comment expliquer qu'une cautérisation puisse laisser des marques colorées comme celles d'Ötzi ? C'est que, pour L. Capasso, celle-ci n'est pas une simple brûlure :

« [...] jusqu'à la fin du siècle dernier, certaines tribus nomades du Tibet pratiquaient " un tatouage thérapeutique » indiqué dans le soin des douleurs articulaires. Le thérapeute faisait une petite coupure cutanée et à l'intérieur de cette dernière, il mettait des herbes médicamenteuses avec un cautère (instrument doté d'une extrémité métallique plate et d'un manche réfractaire), il brûlait les herbes qu'il avait déposées à l'intérieur de la coupure, puis il appliquait une gaze. La cicatrice finissait par englober la particule carbonée laissée par la combustion des herbes médicamenteuses, et il en résultait une sorte de tatouage $\gg{ }^{37}$

La technique de cautérisation indirecte décrite par R. Rolle a malheureusement été mal interprétée par L. Capasso. Ce dernier a cru que la peau était elle-même garnie (gefüllt) d'herbes. Cette technique, déjà exposée par L. Capasso à plusieurs reprises au cours de l'année $1998^{38}$, fut reprise par la presse ${ }^{39}$ et par l'équipe autrichienne, alors que cette dernière publiait de nouvelles hypothèses sur le cas Ötzi ${ }^{40}$.

\footnotetext{
${ }^{34}$ Meyer, 1988, Figs. 48 et 49 ; Kunzang, 1973, p. 27 (description de la technique).

${ }^{35}$ Capasso, 1993, p. 180: "specialized instruments are used, including burners and various tools for cauterization, such as awls and brands. In the traditional Tibetan medicine that is still practiced today, herbs capable of relieving joint pain, especially back pain, are burned against the skin. The therapy is performed with a cauterizing instrument filled with medicinal herbs, whose incandescent point is placed against the afflicted joint ".

${ }^{36}$ Ibid., p. 180: "It therefore is possible that the tattoos found on the body of the Similaun Man represent traces of the practice of localized, topical cutaneous cauterization for the treatment of osteoarthritis".

${ }^{37}$ Capasso et al., 1999, p. 463.

${ }^{38}$ Entretien publié par le quotidien Alto Adige, dans son édition du 16 janvier 1998, et Capasso, 1998.

${ }^{39}$ Le Figaro, no 16623, 23 janvier 1998, p. 14 (R. Heuze s'entretient avec L. Capasso et paraphrase ce dernier) : « une telle méthode était utilisée par des sorciers tibétains il y a encore deux siècles : ils pratiquaient une petite incision sous la peau, déposaient des herbes médicinales dans la blessure et la cautérisaient au fer rouge, la brûlure des herbes soignant la douleur ». Libération, 14 juillet 1998, Cahier spécial été, p. V : N. Levisalles, envoyée spéciale à Bolzano, apprend de L. Capasso que les tatouages d'Ötzi « sont sûrement les traces d'un traitement médical qui est encore utilisé au Tibet : on pratique une petite incision sous la peau et on y dépose une poudre végétale ».

${ }^{40}$ Dorfer et al., 1999, p. 1024.
} 


\section{3. Ötzi et l'acupuncture}

En 1998, l'équipe autrichienne, sous l'impulsion de Léopold Dorfer, fit intervenir des spécialistes de l'acupuncture qui remarquèrent que, parmi les 15 groupes de traits dont était tatoué Ötzi, neuf se trouvaient à proximité de points d'acupuncture chinoise ${ }^{41}$. Cette contiguïté topographique permettrait de considérer la puncture des tatouages d'Ötzi comme l'ancêtre direct de l'acupuncture ${ }^{42}$. Quelques mois après la parution de ces deux articles, deux membres de l'équipe autrichienne (E. Egarter et K. Spindler) amendèrent légèrement leurs conclusions en imaginant cette fois que les tatouages d'Ötzi représentaient « probablement des points de repère pour une espèce d'acupuncture préhistorique », et que «l'on a [vait] donc, à l'origine, utilisé les tatouages pour pratiquer l'acupuncture » ${ }^{43}$.

La topographie des points d'insertion à laquelle ces spécialistes se réfèrent provient du standard que la Chine populaire a diffusé dans ses universités et vers l'Occident depuis $1978^{44}$. La pratique actuelle recense 670 points répartis symétriquement sur tout le corps humain, le long de 12 méridiens (ou canaux) bilatéraux et de deux méridiens axiaux ${ }^{45}$. La surface du corps humain étant littéralement constellée de points d'insertion, on peut estimer comme dépourvu de toute espèce de signification statistique le fait que les tatouages d'Ötzi, longilignes et assez étendus, coïncident de temps à autre avec certains de ces points.

\section{Repères historiques et ethnographiques}

\subsection{L'acumoxa et les autres stimulations thérapeutiques en Chine ancienne}

L'équipe autrichienne a négligé les récents travaux d'histoire de la médecine chinoise, une discipline qui connaît une révolution depuis la découverte, en 1973, des manuscrits médicaux de Mawangdui (tombe $n^{0} 3$, Changsha, province du Hunan) ${ }^{46}$. Ces documents, précisément datés de 168 avant J.-C., constituent la documentation la plus ancienne disponible à ce jour sur la médecine chinoise. Dans cette compilation éclectique se côtoient

${ }^{41}$ Dorfer et al., 1998. L. Capasso et ses collaborateurs ont vraisemblablement mal compris ce court article, puisqu'ils ont cru qu' « une étude récente [...] a [vait] mis en évidence la présence de certains tatouages semblables à de petits points superficiels localisés sur des points traditionnels de l'acupuncture chinoise » (Capasso et al., 1999, p. 463). Or Léopold Dorfer et ses collègues ne prétendent nullement avoir repéré de nouveaux tatouages, mais que «plusieurs d'entre ceux qui pourraient avoir entraîné une puncture superficielle de la peau (that would have entailed superficial skin puncture) semblent être localisés sur des points d'acupuncture chinoise ».

${ }^{42}$ Dorfer et al., 1999, p. 1025.

43 Émission Archimède (Arte, le 3 oct. 2000). Propos retranscrits à l'adresse Internet suivante : http://www.arte.de/hebdo.archimed/20001010/ftexte/sujet8.html.

${ }^{44}$ Pour l'historiographie de la prétendue TCM (Traditional Chinese Medicine), telle qu'elle a été diffusée en Occident, cf. Unschuld et al., 2001, p. 93. Dorfer et al. (1999) utilisent Essentials of Chinese Acupuncture, compiled by Beijing, Shanghai and Nanjing College of TCM, Beijing, 1980. L'un des contributeurs aux deux articles cités, Frank Bahr, membre de l'Académie allemande d'acupuncture et d'auriculomédecine (Munich), est aussi l'auteur de Einführung in die wissenschaftliche Akupunktur, Munich, 1996.

${ }^{45}$ Voir l'exposé des points d'acupuncture par l'Institut de Médecine traditionnelle chinoise de Pékin (Zhang et Wu, 1992).

${ }^{46}$ Récemment traduits et commentés par Donald J. Harper (Harper, 1998). 
recettes médicamenteuses (potions et cataplasmes), stimulations chirurgicales ${ }^{47}$ (cautérisation, saignée, ventouses, compresses chaudes, fumigations, bains, massages), diététique, gymnastique corporelle et pratiques sexuelles, formules d'exorcisme et de magie. D'autres textes médicaux de la première moitié du $\mathrm{II}^{\mathrm{e}}$ siècle avant J.-C. ont également été retrouvés en 1983 dans la tombe $\mathrm{n}^{\circ} 247$ de Zhangjiashan (Jiangling, province du Hubei). Outre des traités inédits, les manuscrits de Zhangjiashan offrent des recensions nouvelles de certains textes de Mawangdui ${ }^{48}$. Enfin, en 1993, dans la tombe $n^{\circ} 2$ de Yongxing (Mianyang, province du Sichuan), datée du II $^{\mathrm{e}}$ siècle avant J.-C., des archéologues ont dégagé ce qui constitue la plus ancienne figurine anatomique chinoise connue à ce jour ${ }^{49}$. Tous ces documents reflètent la diversité de la pensée médicale à l'époque des Han occidentaux et permettent de mesurer le chemin parcouru jusqu'à l'apparition des grands classiques médicaux dont la compilation ne commence qu'au début de notre ère ${ }^{50}$.

Les compilations chinoises classiques inscrivent le corps humain dans un réseau de 12 canaux (mai) bilatéraux où circule le qi (souffle, pneuma). Sur chaque canal sont disposés un certain nombre de points dont les noms et la localisation sont toujours utilisés par les praticiens actuels. La maladie est définie comme un défaut de circulation du qi susceptible d'être corrigé par la puncture ou la cautérisation de certains points spécifiques. Cette méthode de traitement par acupuncture et moxibustion ${ }^{51}$ reçoit parfois l'appellation commode d'acumoxa.

Comme nous allons le voir, les documents exhumés depuis 1973 montrent que les Han occidentaux ne disposaient pas encore d'une topographie et d'une nomenclature standardisée des points d'acumoxa. Les lignes peintes en rouge sur la figurine en bois laqué de Yongxing (neuf lignes bilatérales et une ligne médiane reliant les extrémités des membres à la tête) annoncent sans aucun doute le système ultérieur à 12 mai, bien que leurs trajets ne soient pas toujours comparables à ceux que donneront plus tard les classiques médicaux ${ }^{52}$. Mais, contrairement aux modèles anatomiques en bronze de l'époque médiévale, la figurine de Yongxing ne comporte aucune indication de points d'acumoxa. C'est aussi le cas des textes théoriques de Mawangdui et de Zhangjiashan, qui décrivent les trajets de 11 canaux bilatéraux Yin ou Yang. Chaque mai est chez eux rendu responsable de divers

\footnotetext{
${ }^{47}$ Selon l'acception première de cheirourgia, « pratique manuelle ».

${ }^{48}$ Vivienne Lo prépare actuellement la traduction des textes de Zhangiiashan dont elle a déjà commenté les extraits les plus significatifs (cf. He et Lo, 1996 ; Lo, 2001 ; Lo, 2002).

${ }^{49}$ Décrite et analysée par He et Lo, 1996.

${ }^{50}$ Le classique de référence est le Huangdi neijing (Canon interne de l'Empereur jaune), où un empereur mythique du $\mathrm{III}^{\mathrm{e}}$ millénaire avant notre ère dialogue avec ses conseillers, compilation régulièrement révisée pendant tout le moyen-âge, et dont les matériaux les plus anciens remontent aux $\mathrm{II}^{\mathrm{e}}-\mathrm{I}^{\mathrm{er}}$ siècle avant notre ère. Ce corpus compte trois recensions : le Taisu (Fondements essentiels), le Suwen (Questions élémentaires) et le Lingshu (Pivot de l'Esprit). Les deux autres classiques d'acupuncture et de moxibustion sont : le Nanjing (Classique des difficultés, $\mathrm{I}^{\mathrm{er}}$ ou $\mathrm{II}^{\mathrm{e}} \mathrm{s}$. apr. J.-C.) et, de Huangfu Mi, le Zhenjiu jiayijing (ABC d'acumoxa, entre 256 et 282).

${ }^{51}$ Le terme moxa a été forgé par les voyageurs européens du XVII ${ }^{\mathrm{e}}$ siècle d'après le japonais mo-gouça, lui-même contraction de moé-kouça (« herbe-qui-brûle ») - cf. Huard et Wong, 1958, p. 23. Il désigne le combustible le plus couramment utilisé en Chine et au Japon (un petit rouleau constitué de feuilles d'armoise pilées et enroulées dans une feuille de riz). Dans les lignes qui suivent, moxa et moxibustion désigneront les techniques de cautérisation par combustion organique, distinctes de la cautérisation chimique ou par fer ardent.

${ }^{52}$ Convergences et divergences recensées par He et Lo, 1996, p. 93 sq.
} 
maux affectant les organes et les membres répartis sur son parcours. L'un de ces textes $^{53}$ recommande, comme remède systématique à l'une ou l'autre de ces affections, la cautérisation du canal concerné, sans toutefois préciser le lieu exact où celle-ci doit être pratiquée. Un autre texte ${ }^{54}$ se contente d'établir la liste des canaux et des maux afférents, sans prescrire d'intervention. Il mentionne toutefois les précautions à prendre pour la cautérisation du petit canal Yin ${ }^{55}$. Parce que la cautérisation allait sans doute de soi, le copiste de ce texte n'aura sans doute pas jugé nécessaire de la rappeler après la description de chaque canal $^{56}$. Quoi qu'il en soit, ces textes ne localisent aucun point de moxibustion et ne mentionnent pas la puncture avec aiguille métallique ${ }^{57}$. Vivienne Lo a toutefois attiré l'attention sur un passage du Maishu de Zhangjiashan qui prescrit des cautérisations et des punctures en lien avec le système des canaux :

«Les sages tiennent les canaux en grande estime. Quant au qi, il avantage la partie inférieure du corps et nuit à la partie supérieure; il suit le chaud et s'écarte du froid. Les sages refroidissent donc la tête et réchauffent les pieds. Ceux qui soignent la maladie retranchent l'excédent et comblent le déficit. Si donc le qi monte au lieu de descendre, repère le canal qui a outrepassé ses propres capacités et applique une cautérisation là où il rencontre une articulation. En cas de maladie aiguë, applique une autre cautérisation, deux pouces au-dessus de l'articulation. Si le qi a tendance à monter, puis à redescendre, perfore le [canal] avec une lancette de pierre à l'intérieur du genou et au cou-de-pied $\gg .58$

Cette ordonnance n'est pas sans rappeler certaines interventions qu'un texte du début du $\mathrm{I}^{\mathrm{er}}$ siècle avant J.-C. attribue à Chunyu Yi († c. 150 av. J.-C.), un médecin qui utilisait des lancettes de pierre $^{59}$. Mais cette proto-acupuncture lithique d'époque $\mathrm{Han}^{60}$, de même que la moxibustion, ne semblent pas encore avoir été élevées au rang de thérapeutique géné-

${ }^{53}$ Mawangdui, ms. 1, texte A, tr. Harper, 1998, p. 192-202.

${ }^{54}$ Mawangdui, ms. 1, texte B, tr. Harper, 1998, p. 203-212. Ce texte se trouve également inséré dans le Maishu (Livre des mai) de Zhangjiashan, transcrit sur lattes de bois (lattes 16-47).

55 Harper, 1998, p. 211.

${ }^{56}$ Nous ne suivons donc pas He et Lo (1996 : p. 122) qui, après avoir affirmé que ce traité n'impliquait pas de cautérisation, veulent le rattacher aux seuls exercices de gymnastique thérapeutique.

${ }^{57}$ Paul Ulrich Unschuld a été un des premiers à essayer de tirer les conséquences de ce silence (Unschuld, 1985, p. 93 sq.).

${ }^{58}$ He et Lo, 1996, p. $114=$ Lo, 2001, p. 29. Le texte correspondant de Mawangdui (ms. 1, texte C, Harper, 1998 : p. 213-214) demande de perforer le coude (zhou) au lieu du cou-de-pied ( $f u$ ).

${ }^{59}$ Sima Qian (c. 146-86 av. J.-C.), Shiji (Mémoires historiques), ch. 105 : sur les 18 thérapies mises en œuvre par Chunyu Yi, la plupart sont médicamenteuses, et trois seulement entendent exercer une contrainte sur la circulation du qi dans les vaisseaux : a) défaillance de la terminaison du vaisseau Yin dans le pied, nécessitant la cautérisation du vaisseau en question; b) pour traiter un « retour de chaleur » (re jue) qui échauffe le pied, Chunyu Yi perfore la plante du pied avec trois aiguilles de pierre ; c) pour des maux de tête, un échauffement et un affaiblissement général, Chunyu Yi passe de l'eau froide sur le front de son patient et perfore le canal Yang brillant du pied à trois reprises (localisation non-précisée), afin de contrecarrer un « retour vers le haut » (jue shang) (Harper, 1998 : p. 94 ; Lo, 2002 : p. 109-110).

${ }^{60}$ Vivienne Lo (Lo, 2002 : p. 122) pense avec raison que les neuf aiguilles d'or et d'argent retrouvées en 1968 à Mancheng dans la tombe d'un des membres de la famille impériale (Liu Sheng, † 113 av. J.-C., cf. Hoizey, 1988 : p. 58 ; Harper, 1998 : p. 92) n'étaient pas destinées à l'acupuncture, mais à la couture. Elles ont en effet un chas, et dans la tombe se trouvaient avec elles deux linceuls constitués de morceaux de jade cousus avec du fil d'or. 
rale $^{61}$. On se rend compte en effet, à parcourir les textes traduits par D.J. Harper, que la puncture et la moxibustion ne sont que des moyens de stimulation parmi d'autres, et que leur application (proximale ou distale) obéit rarement à la topographie formalisée des canaux. Pour une même affection, le Wushier bingfang ${ }^{62}$ recourt certes parfois aux stimulations chirurgicales (cautérisation, perforation, compresses chaudes), mais à côté d'autres procédés tels que la pharmacopée, les régimes, les exorcismes et la magie. Donald J. Harper ${ }^{63}$ recense quatre formes de stimulation calorique utilisées dans ce traité en plus de la moxibustion :

- pression chaude ${ }^{64}$;

- rôtissage ${ }^{65}$;

- fumigation ;

- bains chauds.

Les tumeurs à l'aine donnent lieu, dans le Wushier bingfang, à de nombreuses recettes. Outre des formules magiques et des séances d'exorcisme ${ }^{66}$, on prescrit de gratter la tumeur à 14 reprises avec le majeur ${ }^{67}$, de cautériser le tibia ${ }^{68}$ ou bien d'appliquer un moxa au sinciput :

«Prends du reliquat de chanvre et roules-en dans de l'armoise. Utilise ce [moxa] pour cautériser le sommet du crâne d'une personne souffrant d'une tumeur inguinale. Fais-le cloquer, sans plus ». ${ }^{69}$

Une autre recette, lacunaire et difficile à interpréter, mêle perforation et cautérisation :

« Autre. Tumeur inguinale. Lève d'abord les testicules et rabaisse la peau. Perfore le côté du nombril avec une lancette de pierre ... liquide et saindoux ... mélange dans de l'alcool pur. En outre, cautérise la plaie. Tiens-la à l'abri du vent. Pour un meilleur résultat, cautérise le grand [canal] Yin et le grand [canal] Yang ... Excellent ». ${ }^{70}$

La situation à l'époque des Han occidentaux est donc la suivante : les praticiens commencent certes à fonder leurs interventions sur un réseau théorique de canaux, mais

${ }^{61}$ Cf., au II ${ }^{\mathrm{e}}$ siècle avant J.-C., la biographie de Bian Que (IV ${ }^{\mathrm{e}}$ s. av. J.-C.) par Sima Qian, où les potions et les pressions chaudes sont réservées aux affections siégeant à la surface de la peau, tandis que la puncture lithique est censée atteindre les affections plus profondes du sang et des canaux (Lo, 2002 : p. 110).

${ }^{62}$ Prescriptions pour cinquante-deux maladies, Mawangdui, ms 1, texte E, trad. Harper, 1998, p. $221-304$.

${ }^{63}$ Harper, 1998, p. 95.

${ }^{64}$ On utilise souvent un galet trempé dans un liquide chaud (Lo, 2002 : p. 112 sq., qui recherche certains de ces galets parmi les trouvailles archéologiques). D’autres matériaux peuvent également être utilisés (sur une plaie, on applique du sel grillé et enveloppé dans un morceau de tissu ou des boulettes de déjections de ver de terre mélangées avec du vinaigre et chauffées, cf. Wushier bingfang, § 19 et $§ 31$, tr. Harper, 1998 : p. $229-230$ et 235-236).

${ }^{65}$ Un patient qui souffre de rétention d'urine doit tourner son dos vers une flambée de foin et de brindilles, pendant que deux personnes frottent ses fesses (avec un onguent ?) (Wushier bingfang, § 104, tr. Harper, 1998: p. 256).

${ }^{66}$ Cf. Lo, 2002, p. 104.

${ }^{67}$ Wushier bingfang, § 131, tr. Harper, 1998, p. 265.

${ }^{68}$ Ibid., § 140, p. 269.

${ }^{69}$ Ibid., § 127, p. 263.

${ }^{70}$ Ibid., § 135, p. 267. 
sans toutefois disposer encore d'une cartographie bien établie des points de stimulation. Ils héritent d'une thérapie composite où se côtoient divers procédés ${ }^{71}$, parmi lesquels la moxibustion et la puncture, deux techniques qui finiront par acquérir la première place dans les compilations classiques du début de notre ère. Outre son action stimulante, la perforation du tégument répondait parfois à la simple nécessité d'évacuer les poches purulentes des abcès ${ }^{72}$. Dans les classiques médicaux, l'acupuncture se confond même parfois avec la saignée : l'effusion de sang est notamment requise pour réduire une pléthore de qi ou évacuer un agent pathogène ${ }^{73}$. La formalisation du réseau d'acumoxa et l'exploitation thérapeutique de ce dernier sont en Chine les conséquences d'une homogénéisation de l'anatomie humaine qui pourrait avoir pris un virage décisif après la fondation de l'Empire (221 av. J.-C. $)^{74}$, alors que s'instaure un État centralisé soucieux d'harmoniser écriture, poids, mesures, monnaie et code législatif. C'est en effet à partir de cette époque que les élites perfectionnent et formalisent une topographie canonique de canaux et de points d'acumoxa dont l'acupuncture actuelle a hérité.

L'erreur méthodologique de l'équipe autrichienne réside donc dans le fait d'avoir pensé pouvoir retrouver sur la peau tatouée d'Ötzi une topographie de points d'acumoxa qui est de toute évidence le fruit d'un consensus culturel largement postérieur. Si nous considérons comme invalide la démonstration topographique de Léopold Dorfer et al., nous sommes en revanche prêts à reconnaître la haute antiquité des interventions de petite chirurgie comme la saignée, la cautérisation, le tatouage, et même la puncture pratiquée avec ou sans matière colorante, telle qu'elle a survécu chez des peuples d'Asie septentrionale.

\subsection{Puncture et perforation chez les peuples arctiques}

Plusieurs parallèles ethnographiques peuvent être versés au dossier des origines de l'acupuncture chinoise. Ils sont à rechercher du côté des thérapeutiques inuits. Robert Fortuine a répertorié la plupart des observations sur la chirurgie arctique, collectées depuis les premières explorations de Vitus Behring ${ }^{75}$. Son article montre que, parmi les diverses thérapies chirurgicales existantes, une place privilégiée a été donnée au procédé consistant à transpercer la peau avec une lancette de pierre. Ces perforations offrent des analogies frappantes avec la puncture lithique telle qu'elle nous est apparue à travers les documents chinois du $\mathrm{II}^{\mathrm{e}}$ siècle avant notre ère.

C'est à un célèbre missionnaire russe des îles aléoutiennes, le Père Veniaminov ${ }^{76}$, que l'on doit les meilleures informations sur le sujet. Il remarque qu'en cas de crachats sanguinolents, les médecins aléoutes puncturent le phtisique sous les côtes avec des lancettes de pierre « afin d'évacuer le mauvais sang et le pneuma (духь) vicié ».

\footnotetext{
${ }^{71}$ Vivienne Lo a montré comment des massages ou des exercices de gymnastique thérapeutique pouvaient parfois se conformer au circuit de certains canaux reproduits sur la figurine de Yongxing (He et Lo, 1996 : p. 118-122).

${ }^{72}$ Mawangdui, ms 1, texte C (Maifa), tr. Harper, 1998, p. 215-216.

73 Textes traduits et analysés par Elper, 1980. Inversement, en cas de carence de qi, le ch. 62 du Suwen (Huangdi neijing) demande de laisser l'aiguille plantée un moment dans le vaisseau en attendant qu'il gonfle ou que le pouls s'accélère, puis de la retirer rapidement en prenant soin d'éviter toute effusion de sang (ibid., p. 357).

${ }^{71}$ Sur cette tentative de systématisation rationnelle, cf. Unschuld et al., 2001, p. 31 sq.

${ }^{75}$ Fortuine, 1985.

${ }^{76}$ Ivan Evseïevitch Veniaminov (1797-1879). Il fut canonisé sous son nom monastique, Innokenti.
} 
«Cette opération n'est toutefois accomplie que par les médecins les plus expérimentés, car elle exige de connaître dans le détail où et à quelle profondeur il faut puncturer et quelle quantité de pneuma doit être évacuée. Sinon, on risque d'endommager les organes internes et d'expédier le patient dans l'autre monde en laissant échapper tout le pneuma ${ }^{77}$

Cette puncture thoracique est utilisée pour d'autres maladies internes (les coliques en particulier) et comme traitement de la dernière chance. Mais Veniaminov mentionne aussi d'autres types de puncture :

« [...] la puncture est aussi employée pour beaucoup d'autres maladies. Par exemple, pour des douleurs oculaires, on perfore la peau entre les yeux après l'avoir soulevée de l'os, ou bien on perfore au niveau des tempes, de la nuque, etc. En somme, il n'y a pas d'endroits sur le corps, à l'exception des yeux eux-mêmes, qui ne soient susceptibles d'être puncturés en cas de maladie. J'ai connu un Aléoute qui avait été puncturé plus de quarante fois à différents endroits $\gg{ }^{78}$

La perforation consistant à soulever un pli de la peau et à le transpercer de part en part est caractéristique des régions arctiques. Nous l'avons trouvée mentionnée sous la plume de Georg Wilhelm Steller, qui explora le Kamtchatka vers 1740 :

«Lorsque les pieds leur font mal, [les Kamtchadales] prennent la peau autour de la cheville avec deux petits morceaux de bois, la soulèvent vers le haut, la transperçent avec une lancette de cristal (Messerlein von Crystal gemacht) et laissent s'écouler autant de sang qu'il peut en sortir ; et ils disent que ce procédé leur a souvent procuré un soulagement ${ }^{79}{ }^{79}$

D'après R. Fortuine, les Inuits de l'île de Nunivak et de Nunamiut pratiquent cette perforation autour d'une articulation douloureuse et/ou enflée. On se contente parfois d'une simple incision à proximité de la zone affectée (sur le crâne pour une céphalée, autour des yeux pour des douleurs oculaires, sur le dos ou à côté du genou pour un rhumatisme $)^{80}$. Une autre variante consiste à introduire une matière colorante, en faisant passer sous la peau un fil teinté de noir de fumée. Lars Krutak a encore récemment observé ce procédé sur l'île Saint-Laurent, où il est surtout utilisé à proximité des articulations. D'après lui, le passage du fil laisse deux points noirs sous la peau ${ }^{81}$. Selon d'autres observateurs, cette méthode dessine des petits traits :

\footnotetext{
77 Veniaminov, 1840 (1984), p. 292.

78 Ibid., p. 293.

${ }^{79}$ Steller, 1774 , p. 366.

${ }^{80}$ Fortune, 1985, p. 33-34.

${ }^{81}$ Krutak, 1999.
} 
"Sur l'île d'Atka, en cas de douleurs articulaires ou dorsales, la peau des zones affectées est pincée et soulevée, puis cousue avec un fil imprégné de poudre à canon (autrefois probablement imprégné de suie) qui laisse des lignes noires $\gg{ }^{82}$

Cette technique est conforme au procédé souvent employé pour le tatouage ornemental en Arctique et en Sibérie orientale ${ }^{83}$ à côté de la puncture simple. Des tatouages thérapeutiques analogues ont été signalés par S.I. Rudenko chez les Inuits de la presqu'île des Tchouktches :

«Le tatouage non-ornemental (незорная татуировка) - le tatouage à lignes simples (татуировка простыли линиями) - était mis et est mis encore maintenant sur les membres malades (назаболевшие члены) dans un but thérapeutique (с медичинской целью), tant par les femmes que par les hommes ». ${ }^{84}$

L'emploi thérapeutique de la puncture chez les peuples arctiques n'est pas la seule analogie susceptible d'être établie avec l'ancienne médecine chinoise. G.H. Marsh et W.S. Laughin avaient jadis remarqué une convergence de vues entre les conceptions anatomiques traditionnelles des Aléoutes et celles de la médecine chinoise ${ }^{85}$. Ce dossier mériterait d'être réexaminé à la lumière des documents Han occidentaux récemment mis au jour. Le fait que la puncture soit parfois confondue chez les Inuits avec le tatouage thérapeutique pose la question de l'antériorité de ce dernier sur l'acupuncture chinoise. On ferait néanmoins fausse route en prétendant donner à chaque type de mutilation thérapeutique une place déterminée sur un arbre généalogique. Nos sources attestent seulement, sans plus de précisions, la haute antiquité et la large diffusion de certains procédés. C'est sans doute le cas de la puncture simple, bien que notre documentation, essentiellement asiatique, ne nous permette pas de remonter plus haut que le $\mathrm{II}^{\mathrm{e}}$ siècle avant notre ère. C'est indéniablement le cas du tatouage, depuis l'étonnante découverte d'Ötzi. Restent d'autres pratiques moins généreuses en vestiges, mais peut-être tout aussi anciennes, comme les massages, les fumigations, les bains, ou les cataplasmes ${ }^{86}$.

Notre excursus du côté de la Chine nous a permis de réviser l'image hiératique et intemporelle que certains praticiens modernes voudraient donner de l'acupuncture. Avant que les grands classiques médicaux ne la mettent au centre de leur thérapeutique et

\footnotetext{
${ }^{82}$ Lantis, 1984, p. 174.

${ }^{83}$ Johann Georg Gmelin assista en janvier 1738 à la « couture » d'un tatouage toungouze qu'il décrit en détail dans son récit de voyage - cf. Gmelin, 1751-1752, vol. 2, p. 649-650 et aussi p. 207-208 (description des tatouages). En revanche, en Sibérie occidentale, le tatouage des Ougriens de l'Ob (Ostiaks, Khants-Mansi) se fait par puncture, comme partout ailleurs en Occident.

${ }^{84}$ Rudenko, 1949a, p. 149.

${ }^{85}$ Marsh et Laughin, 1956, p. 54 : "it seems plausible that both the Aleuts, together whith other Alaskan Eskimo groups, and the Chinese have continued a tradition going back to a common Mongoloid ancestral people in northeastern Asia".

${ }^{86}$ Ces pratiques sont abondamment illustrées par les prescriptions magico-médicinales des textes cunéiformes (Herrero, 1984). Ces archives n'ont, dans l'état actuel de nos connaissances, presque rien donné concernant la petite chirurgie. Voir toutefois les deux saignées aux tempes en cas de céphalée chez Attia et Buisson, 2003, p. 13, 1. 64 et p. 14, 1. 180-186. Nombreux onguents et cataplasmes également dans la médecine égyptienne (Bardinet, 1995).
} 
l'ajustent à une topographie canonique du corps humain, d'autres documents nous la font voir sous un jour très différent : l'aiguille métallique n'a pas encore détrôné la lancette de pierre, l'opération peut être sanglante, le réseau anatomique connaît encore des variations dans le parcours de ses canaux, et les points d'insertion qui dépendent de celui-ci ne sont pas encore définitivement fixés. Or, si la topographie des points d'acumoxa formalisée au début de notre ère n'est déjà plus comparable à celle qui avait cours au $\mathrm{II}^{\mathrm{e}}$ siècle avant, il y a d'autant moins de raisons de chercher à la superposer à celle des tatouages d'Ötzi.

\subsection{Le tatouage thérapeutique en Eurasie et en Méditerranée}

Les parallèles ethnographiques que nous devons privilégier sont évidemment ceux qui nous renseignent sur les emplois thérapeutiques du tatouage. Dans le cas d'Ötzi, ces parallèles ont été jusqu'à présent négligés, et nous souhaitons ici en étoffer l'inventaire. De fait, les interventions punctiformes ou linéaires que nous avons constatées chez les Inuits ne sont pas isolées : d'autres attestations de cette thérapeutique élémentaire apparaissent dans des régions beaucoup plus proches d'Ötzi.

Si nous poursuivons vers l'Ouest le chemin que nous avons commencé sur les rives de la mer de Béring, il nous faut normalement faire halte à nouveau dans l'Altaï. Nous n'ajouterons rien à ce que nous avons dit plus haut sur l'Homme de Pazyryk, sinon que les points qu'il porte tatoués sur le dos et à la cheville semblent maintenant offrir des analogies frappantes avec les interventions punctiformes inuit ${ }^{87}$. En Sibérie occidentale, les Ougriens de l'Ob (Khants-Ostiaks et Mansi-Vogouls) utilisèrent jusqu'au début du $\mathrm{XX}^{\mathrm{e}}$ siècle des tatouages thérapeutiques constitués de petits traits horizontaux ${ }^{88}$. Beaucoup plus rarement, il semble que certains motifs géométriques empruntés au répertoire tatoué ornemental (essentiellement féminin) aient été employés dans un but thérapeutique ${ }^{89}$.

Les rapports britanniques du début $\mathrm{du} \mathrm{XX}^{\mathrm{e}}$ siècle consacrés au tatouage des tribus nomades ou semi-nomades de l'Inde se sont forts peu intéressés au tatouage thérapeutique $^{90}$. Edgar Thurston l'a pourtant observé en Inde méridionale :

«Chez les femmes indigènes, les parties du corps dévolues au tatouage sont les bras, les jambes, le front, les joues et le menton. Mais parfois, en cas de douleurs musculaires ou autres problèmes, le tatouage est exécuté comme un soin thérapeutique

\footnotetext{
${ }^{87}$ On peut se demander si les points qui à Pazyryk se font face de part et d'autre de la colonne vertébrale n'auraient pas pu être obtenus en passant une aiguille à travers un large pli de peau que l'on aurait soulevée juste au-dessus du rachis. S.I. Rudenko ne précise pas si les points manquant de la partie droite ont été omis de façon délibérée par le tatoueur ou si leur absence résulte de la dégradation du tégument à ces endroits.

${ }^{88}$ On doit à un savant finnois (Arturri Kannisto, 1933), qui enquêta lui-même sur le sujet en Sibérie dans les années 1902-1906, un inventaire très exhaustif des rares relevés et commentaires publiés depuis le début du XVIII ${ }^{\mathrm{e}}$ siècle sur le tatouage des Ob-ougriens.

${ }^{89}$ Natalia V. Polosmak prétend que les Khants-Mansi auraient eu coutume de transférer certaines affections internes vers des tatouages zoomorphes (mammifères ou oiseaux, cf. Polosmak, 2000 : p. 101). Cette assertion, déjà exprimée en 1921 par K.F. Karjalainen, a été réfutée de façon convaincante par A. Kannisto (Kannisto, 1933 : p. 175-177).

${ }^{90}$ Silence total chez Gupte et Rose, 1902 et chez Luard, 1904.
} 
(remedial agent) sur l'articulation de l'épaule, sur la cuisse, ou sur d'autres endroits du corps $\gg .^{91}$

Chez les femmes Kol du centre de l'Inde, on tatoue également des petits traits et des points sur le siège d'une douleur, ou même sur les seins pour favoriser la production de lait $^{92}$.

Les tribus indiennes pratiquent une forme de tatouage ornemental féminin qui possède des analogies stylistiques avec le tatouage féminin des tribus irakiennes. Or l'Irak lui non plus n'ignore pas le tatouage thérapeutique, comme nous allons le voir en nous rapprochant maintenant du Bassin méditerranéen.

De nombreuses observations sur l'emploi du tatouage thérapeutique ont été recueillies auprès des populations berbères, bédouines ou arabes établies en Afrique du Nord et au Proche-Orient. Ces données sont, pour le cas Ötzi, du plus haut intérêt ${ }^{93}$. Nous avons réuni ces données sous trois rubriques : Irak, Égypte et Maghreb.

\subsubsection{Irak}

Parmi les nombreux tatouages ornementaux relevés dans les années 1930 par Henry Field en Irak ${ }^{94}$, plusieurs ont une destination thérapeutique : un membre de la tribu des Yezidis, du district de Sheikan (nord de l'Irak), explique que les croix et les points de sa cheville gauche, comme le cercle de sa cheville droite, ont été tatoués pour traiter le rhumatisme ${ }^{95}$. Les hommes, souvent tatoués au poignet de motifs géométriques (parfois cruciformes), invoquent parfois un rhumatisme, une foulure, ou simplement la nécessité de renforcer le poignet ${ }^{96}$. À l'une des ethnologues de l'équipe d'H. Field, qui avait déjà remarqué le fréquent recours au tatouage contre les entorses du poignet, un tatoueur professionnel confirmera que le tatouage est effectivement destiné à le renforcer ${ }^{97}$. Henry Field a également rassemblé un grand nombre de cas de tatouage temporaux indiqués contre les maux de tête ${ }^{98}$, analogues à ceux enregistrés en Égypte par D. Fouquet, et qui peuvent à l'occasion être cruciformes ${ }^{99}$. En 1961, Maria Czapkiewicz pouvait encore

\footnotetext{
${ }^{91}$ Thurston, 1906, p. 380.

92 Griffiths, 1946, p. 29.

${ }^{93}$ L'équipe néerlandaise a pu noter : « La localisation et la forme [des tatouages d'Ötzi] montrent une remarquable ressemblance avec les tatouages thérapeutiques que l'on peut voir de nos jours en Inde et en Afrique » (Van der Velden et al., 1995 : p. 277). De son côté - mais sans davantage de précisions - Konrad Spindler n'ignore pas que « chez les Berbères d'Afrique du Nord, le tatouage des parties douloureuses du corps est une pratique bien établie » (Spindler, 1997 : p. 19).

94 Field, 1958. Le tatouage ornemental irakien est essentiellement féminin et constitue un intermédiaire stylistique entre le tatouage indien et celui d'Afrique du Nord.

95 Ibid., p. $20, \mathrm{n}^{\mathrm{o}} 2437$.

96 Ibid., p. 20 ( $\mathrm{n}^{\mathrm{o}}$ 2402, 2417, hommes Yezidis, pour renforcer le poignet) ; p. 23 ( $\mathrm{n}^{\mathrm{o}}$ 1985, Kurde, rhumatisme) ; p. 27 (deux hommes Sulubba, foulure), etc.

${ }^{97}$ Smeaton, 1937, p. 58.

${ }^{98}$ Field, 1958, qui note p. $14:$ : le tatouage aux tempes [...] est ordinairement exécuté comme remède au mal de tête ». Le rapport d'Henry Field se contente souvent de signaler le tatouage temporal pour les divers groupes étudiés, sans préciser à chaque fois son indication thérapeutique.

99 Ibid., p. 42 : un arabe Marsh tatoué d'une croix sur chaque tempe au niveau des sourcils, et d'une file de points à la base du front (Fig. 31, no $871=$ notre pl. VIII, Fig. 7). Autres observations faites en 1950 en Iran : un
} 
observer des tatouages thérapeutiques en Irak, tout en notant une progressive désaffection de la pratique ${ }^{100}$.

\subsection{2. Égypte}

La première collecte de tatouages thérapeutiques en Égypte est due au Dr Daniel Fouquet, qui rassembla à la fin du $\mathrm{XIX}^{\mathrm{e}}$ siècle des observations effectuées auprès d'Égyptiens des classes populaires ${ }^{101}$ : en tête de liste des affections traitées par le tatouage vient la céphalée, à laquelle on tente de remédier au moyen d'incisions sur les tempes (60 cas répertoriés), souvent teintées au noir de fumée. Ces tatouages prennent le plus souvent la forme de trois incisions verticales surmontées parfois de trois points disposés en triangle $^{102}$. Employés indifféremment par les Coptes ou les Musulmans, ces tatouages ne s'observent que parmi les classes populaires. D. Fouquet enregistre également 23 cas de tatouages indiqués pour des problèmes articulatoires ou osseux, constitués de points ou de traits parallèles - parfois inscrits dans un petit quadrangle -, et placés aux endroits douloureux. On tatoue ainsi le poignet, le dos de la main et les phalanges ${ }^{103}$, mais aussi le genou $^{104}$, la cheville ${ }^{105}$ et le pied ${ }^{106}$. Quatre cas de tatouages linéaires (lignes brisées) ont par ailleurs été relevés sur l'abdomen de femmes coptes originaires de Haute-Égypte, pour traiter des maux de ventre ${ }^{107}$. Enfin, contre des grosseurs apparues à la base du cou, D. Fouquet note des tatouages de forme circulaire, appliqués à deux reprises sur l'enflure elle-même. Les dernières occurrences signalées par D. Fouquet concernent des maladies de peau que l'on a cherché à dissimuler derrière un tatouage ${ }^{108}$.

\subsubsection{Maghreb}

Le tatouage thérapeutique au Maghreb commence à être enregistré à partir de la fin du $\mathrm{XIX}^{\mathrm{e}}$ siècle. Au cours d'une petite enquête menée auprès d'Algériens de la ville d'Annaba (anciennement Bône), Louis Batut relève trois cas de tatouage thérapeutique : une vieille femme arabe lui montre trois lignes scarifiées au coin de l'œil à la suite d'un traumatisme oculaire survenu lorsqu'elle avait 14 ans. Chez un spahi en garnison, L. Batut relève aussi un tatouage par scarification sur le poignet et le dos de la main, par lignes entrecroisées, indiqué contre une hydarthrose du poignet. Enfin, un tirailleur kabyle présente un tatouage

homme Lurs porte une croix sur chaque tempe ; un Kurde est tatoué d'une croix à la tempe gauche pour des maux de tête (ibid., p. 50).

${ }^{100}$ Czapkiewicz, 1962.

${ }^{101}$ Fouquet, 1898.

102 Ibid., Figs. 3-12.

${ }^{103}$ Ibid., p. 276-277, et figures correspondantes.

${ }^{104}$ Un seul cas de tatouage occupant l'intérieur de l'articulation, composé de quatre traits parallèles et de trois groupes de trois points, chez une femme de 54 ans (Ibid., p. 277-278 et Fig. 21).

${ }^{105}$ Comme traitement pour une entorse chez un Copte du Vieux-Caire, une ligne au-dessous de la malléole externe du pied gauche (Ibid., p. 277 et Fig. 18).

${ }^{106}$ Six points sur la région antéro-externe du pied droit chez un musulman de Tantah, pour traiter une douleur et un gonflement à cet endroit (Ibid., p. 277 et Fig. 19). D. Fouquet signale aussi deux autres tatouages, l'un circulaire et l'autre formé d'incisions parallèles, placés respectivement sur la face externe du pied droit (Musulman de Benha) et à la base du gros orteil droit (Copte du Caire).

107 Ibid., p. 278.

${ }^{108}$ Ibid., p. 278-279. 
à la base du cou censé combattre une douleur interscapulaire ${ }^{109}$. Des faits similaires sont notés à la même époque par Émile Mauchamp (†1907) dans son étude sur les superstitions marocaines :

«Les tatouages sont parfois employés au Maroc - comme il est d'usage en Arabie pour préserver de certaines maladies ou les guérir, ou encore pour écarter quelque menace de mauvais sort. Pour une foulure, on fait, autour du poignet, un tatouage en bracelet serpentin, les courbes dépassant les limites du mal vers la main et vers le bras. On tatoue de même sur les tumeurs, les kystes sébacés. Contre l'hydarthrose, on fait un tatouage sur la partie interne du genou, derrière la rotule $~^{110}$

Les meilleures pages relatives au tatouage thérapeutique au Maghreb se lisent dans une thèse de médecine, soutenue en 1940 à l'Université de Médecine d'Alger par René Chabelard, et dans laquelle sont décrits 18 cas de tatouages thérapeutiques ${ }^{111}$. Les conclusions de R. Chabelard, qui rejoignent les observations du Dr Fouquet en Égypte, méritent d'être citées :

«Le tatouage curatif est dirigé contre un symptôme déterminé : il se pratique loco dolenti par piqûre et le plus souvent par scarification : il est ensuite coloré. On le trouve avec la plus grande fréquence sur les membres, dans les régions articulaires et surtout sur les articulations du poignet et du cou-de-pied, où il est dirigé contre les douleurs articulaires, les atteintes des gaines et des tendons. Il est la plupart du temps constitué de lignes entrecroisées et de points formant bracelet autour de la région ${ }^{112}$

Parmi les 16 cas les plus significatifs, sept concernent des affections accidentelles ou rhumatismales du poignet, de la main ou de l'avant-bras ${ }^{113}$; trois concernent les membres inférieurs $^{114}$; deux tatouages ont été pratiqués pour combattre diverses grosseurs ${ }^{115}$; deux

${ }^{109}$ Batut, 1893, p. 82.

${ }^{110}$ Mauchamp, 1911, p. 141-142.

${ }^{111}$ Chabelard, 1940 (l'étude du tatouage thérapeutique occupe les p. 76-96).

112 Ibid., p. 78.

${ }^{113}$ Ibid., observation III (p. 81 et Fig. 8, p. 82 : contre des douleurs articulaires, tatouage par scarification « constitué par de petits quadrilatères disposés en bracelet autour du poignet et couvrant la surface dorsale de la main ») ; obser. VII (p. 86 : contre un rhumatisme aigu au poignet, « tatouage en forme de bracelet surmonté de deux palmes ») ; obser. VIII (p. 87 et Fig. 10c : contre un rhumatisme, « quadrilatère incomplet dont les côtés portent eux-mêmes des appendices verticaux », tatoué à l'aiguille sur le poignet) ; obser. XI (p. 90 et Fig. 11c, p. 89 : à la suite d'une chute sur la main, tatouage par scarification sur le poignet, en forme de losange entrecoupé de lignes et dentelé vers l'extérieur) ; obser. XIV (p. 92 et Fig. 12b, p. 91 : tatouage ornemental - losanges dentelés - par scarification sur un avant-bras qui présentait une atrophie musculaire) ; obser. XV (p. 92 et Fig. 13 a, p. 93 : tatouage ornemental - losanges - par scarification sur le plat supérieur de la main pour une arthrite chronique du poignet) ; obser. XVI (p. 93-94 et Fig. 13b : tatouage à l'aiguille en forme de peigne sur le poignet, prolongé par des lignes dentelées sur le plat supérieur de la main, pour une luxation du poignet).

${ }^{114}$ Ibid., obser. IV (p. 84 et Fig. 9, p. 83 : deux petites croix tatouées par scarification autour de la rotule contre des douleurs au genou) ; obser. XVII (p. 94 et Fig. 14a, p. 95 : lignes dentelées tatouées par scarification autour de la rotule, et autres motifs géométriques sur la rotule elle-même pour une enflure du genou) ; obser. XVIII (p. 95 , Fig. 14b : pour un coup de pied reçu au tibia, deux traits horizontaux dentelés tatoués sur la rotule, tatouages 
autres pour des problèmes gastriques ${ }^{116}$; enfin deux sujets se sont fait tatouer à proximité des yeux pour des affections oculaires ${ }^{117}$.

\section{4. Ötzi et la pratique du tatouage dans l'Antiquité}

On devine sans peine, à la lecture des observations réunies ci-dessus, quels sont les parallèles ethnographiques que nous entendons privilégier. Est-ce à dire qu'Ötzi a traversé la Méditerranée ? Les attestions anciennes du tatouage que nos recherches personnelles nous ont amené à réunir devraient permettre d'éclaircir cette question. Dans l'Antiquité, le

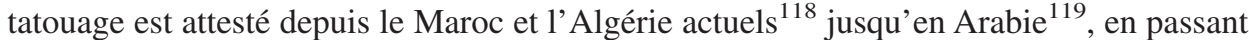
par la Libye ${ }^{120}$ et la Nubie ${ }^{121}$. Désapprouvé par la législation hébraïque (Lv 19, 28), le tatouage se retrouve un peu plus tard en $S_{y}$ rie $^{122}$ et en Palestine ${ }^{123}$, et sur les rives turques de la Mer Noire ${ }^{124}$. Plusieurs notices antiques font état de peuplades tatouées établies au nord de la Mer Noire et dans le bassin du Danube ${ }^{125}$. Parmi elles, les Thraces occupent la première place : les tatouages féminins qui avaient cours chez eux sont mentionnés par de nombreux auteurs ${ }^{126}$, et les vases grecs des $\mathrm{V}^{\mathrm{e}}-\mathrm{IV}^{\mathrm{e}}$ siècles représentent souvent des thraciennes tatouées ${ }^{127}$. Des analogies peuvent même être décelées entre le tatouage thrace

punctiformes sous le genou, enfin, sur le tibia, série de traits verticaux dentelés, terminés par des petites croix, puis zigzags et points).

${ }^{115}$ Ibid., obser. II (p. 80 et Fig. 10a, p. 87 : composition géométrique tatouée sur un goitre du cou) ; obser. IX (p. 88 et Fig. 11a, p. 89 : tatouage réalisé en deux fois autour d'une tumeur blanche du pied : quadrilatère puis ovale).

${ }^{116}$ Ibid., obser. X (p. 89, Fig. 11 b : petits cônes rangés en cercles tatoués par scarification sur le ventre pour aérophagie et constipation intense) ; obser. XIII (p. 91-92, Fig. 12a : composition géométrique tatouée par scarification sur le ventre pour une tachycardie provoquant des battements de l'aorte abdominale).

${ }^{117}$ Ibid., obser. V (p. 85 et Fig. 10b, p. 87 : tatouage circulaire avec croix inscrite sur la tempe gauche pour un glaucome); obser. VI (p. 85 : trois petits traits horizontaux divergents tatoués à l'angle externe de chaque œil pour un trachome).

${ }^{118}$ Scylax (IV e s. av. J.-C.), Périple, 111 (les Aethiopes à peau claire du Maroc actuel sont ornés de tatouages) ; Cassius Félix, La médecine des Grecs (447 apr. J.-C.), 13, 1 et 2 (les femmes maures d'Afrique du Nord sont tatouées).

${ }^{119}$ Plusieurs poètes arabes pré-islamiques utilisent le tatouage comme la métaphore des vestiges laissés par les villages ou les campements abandonnés.

${ }^{120}$ Anciens Téméhou représentés dans l'art égyptien du Nouvel Empire ; peintures plus anciennes du Sahara.

${ }^{121}$ Plusieurs momies féminines tatouées ont été découvertes en Haute-Égypte (c. 2000 av. J.-C.) et en Nubie (c. 1500 av. J.-C. et début de notre ère). Elles montrent qu'un même répertoire décoratif est resté presque inchangé pendant deux millénaires.

${ }^{122}$ Papyrus du Louvre no 2333 (Égypte, 146 av. J.-C.) : un esclave venant de Bambyce-Hiérapolis en Syrie est tatoué au poignet de lettres araméennes. Il pourrait s'agir d'une marque d'esclavage, bien que Lucien de Samosate affirme que tous les Syriens de cette ville sont tatoués au poignet ou au cou (Déesse syrienne, 59).

123 Théodoret de Cyr († c. 460), Questions sur le Lévitique (19, 28).

124 Xénophon (c. 430-354), Anabase, V, 4, 32 (les Mossynèques, établis aux environs de l'actuelle Trébizonde).

${ }^{125}$ Cléarque de Solos (III ${ }^{\mathrm{e}}$ siècle av. J.-C.) ap. Athénée, Deipnosophistes, XII, 524d-e (Scythes et Thraces) ; Pline 1'Ancien, Histoire naturelle, XXII, 2 (Daces et Sarmates) ; Virgile, Énéide, IV, 146 et Pomponius Mela, Chorographie, II, 1, 10 (Agathyrses).

${ }^{126}$ Entre autres : Hérodote, Histoires, V, 6 ; Phanoclès, Amours ou beaux garçons, frag. 1, 23-27 ; Anthologie palatine, VII, 10, 3 ; Dion Chrysostome, Discours 14, 19-20.

127 Zimmermann, 1980. 
apparaissant sur les vases grecs et le tatouage des femmes irakiennes connu par les relevés du début du $\mathrm{XX}^{\mathrm{e}}$ siècle $^{128}$. Nous savons enfin que le tatouage était pratiqué dans l'Antiquité jusque sur les versants occidentaux des Alpes dinariques ${ }^{129}$, donc à quelques encablures du lieu où Ötzi a été retrouvé. Ce tatouage balkanique a peut-être survécu jusqu'à nos jours chez les femmes catholiques en Bosnie-Herzégovine et au nord de l'Albanie ${ }^{130}$. Notons que, contrairement à une idée répandue, l'archéologie et les sources anciennes ne permettent pas d'inférer la pratique du tatouage pour le monde germanique et celte ${ }^{131}$.

La cartographie du tatouage antique que nous venons d'esquisser dessine autour du bassin méditerranéen un vaste croissant ininterrompu depuis le Maroc jusqu'à la Mer Adriatique. Le tatouage y est le fait de communautés sans écriture dont la culture est encore enracinée dans la préhistoire. Les grandes civilisations (Égypte, Mésopotamie, Israël, Grèce et Rome) en sont presque exemptes : le tatouage n'a plus chez elles qu'un rôle utilitaire souvent dégradant (marquage des captifs, des esclaves et des condamnés). La documentation ancienne confirme ce que l'ethnographie a depuis longtemps établi : le tatouage méditerranéen est avant tout une parure féminine. Mais le laconisme des auteurs anciens laisse souvent la question du tatouage thérapeutique de côté. Deux anecdotes tardives permettent toutefois d'en retrouver la trace. La première est extraite de la Vie de saint Porphyre de Gaza ( $\mathrm{V}^{\mathrm{e}} \mathrm{s}$.) : trois jeunes garçons palestiniens tombés dans un puits sont remontés sains et saufs après les prières de Porphyre, or "Tous trois furent découverts portant des signes cruciformes (stauroeidès sèmeia) comme provoqués par une égratignure d'aiguille (ôs apo xesmatos belonès), l'un au milieu du front, l'autre sur la main droite à la naissance des doigts, le troisième à l'épaule droite. Les petites croix étaient bien formées, ni de guingois ni tordues, mais d'un mètre uniforme, de sorte qu'il était manifeste que c'étaient des signes divins (theosèmeia). Elles ne leur faisaient d'ailleurs aucun mal ; mais, sans qu'il y ait de sang, elles semblaient avoir été marquées au cinabre (tetupômena ôs apo kinnabareôs). Elles demeurèrent sur eux assez longtemps pour que tous les vissent et les admirassent : et parmi les païens beaucoup à ce miracle gagnèrent la foi $\gg .{ }^{132}$

Ces petites croix palestiniennes - portées par des enfants qui n'étaient peut-être pas baptisés, et que les pieux spectateurs ont identifiées avec le symbole chrétien salutaire rappellent les divers motifs linéaires, souvent cruciformes, rencontrés dans le répertoire du tatouage thérapeutique. Ces tatouages de couleur rouge, destinés à disparaître progressivement, font aussi penser aux tatouages provisoires que certaines femmes marocaines dessinent sur leurs chevilles ou leurs poignets avec un couteau, puis saupoudrent de

${ }^{128}$ Tatouage du cou et du menton, files de chevrons emboîtés, quadrupède schématique constituant l'exception figurative au sein d'un répertoire exclusivement géométrique.

${ }^{129}$ Strabon, Géographie, VII, 5, 4 (Iapodes et Illyriens).

130 Truhelka, 1896. Les Catholiques bosniaques représentent le substrat ethnique local en partie christianisé par Rome avant l'arrivée au VII ${ }^{\mathrm{e}}$ siècle des Slaves (Serbes). Ces derniers, appelés à devenir orthodoxes, n'étaient toujours pas convertis au $\mathrm{X}^{\mathrm{e}}$ siècle (Constantin Porphyrogénète, Administration de l'Empire, 31).

${ }^{131}$ Certains guerriers germains (Tacite, Germanie, 43, 6) et bretons (César, Guerre des Gaules, 5, 14) se badigeonnaient le corps de noir ou de bleu avant de partir au combat. Ces peintures corporelles ont été confondues dès l'Antiquité avec des tatouages indélébiles.

${ }^{132}$ Marc le Diacre, Vie de Porphyre, évêque de Gaza, 82, éd. et trad. (légèrement modifiée) H. Grégoire et M.-A. Kugener, Paris, 1930, p. 65. 
henné ${ }^{133}$. Il nous semble que les trois enfants de Gaza ont sans doute été marqués de la sorte par leur mère, dans un but prophylactique. Des tatouages infantiles de ce genre sont en effet bien attestés en Afrique du Nord :

«Quand l'enfant marche et peut échapper à la surveillance et à la protection de sa mère, celle-ci lui fait de petits tatouages sur les genoux et autour des chevilles. Ce sont des raies verticales par petits groupes de deux raies parallèles. Ces tatouages gardent jalousement l'enfant de l'envie et du mauvais oeil des génies et des gens ». ${ }^{134}$

Il s'agit d'une scarification "constituée par de petits traits juxtaposés, noircis à la suie, que l'on pratique sur l'un des côtés du nez ou sur le menton, parfois sur le bras. On la fait aux enfants, dans les familles particulièrement éprouvées par la maladie infantile ${ }^{135}$

«Le plus souvent, la ayyacha [phylactère] est constituée par des scarifications que l'on a frottées avec du henné ou du noir de fumée. Ces scarifications sont d'usage courant dans toute l'Afrique du Nord. En Tunisie, les mères les font au menton, à la joue et surtout au front de leur enfant, parce qu'elles " font vivre, elles conservent la vie "». ${ }^{136}$

La seconde anecdote date de 591. Le roi perse sassanide Khosrō II sollicite cette année-là les troupes de l'empereur byzantin Maurice pour mettre en échec la sédition de son rival Bahrām Tchobēn. Le général byzantin Narsès fait un grand nombre de prisonniers. Certains d'entre eux, des Turcs orientaux (ek tou ethnous tôn tourkôn), sont ramenés en triomphe devant l'empereur Maurice :

«L'emblème de la passion du Seigneur était marqué sur les fronts des captifs, cet emblème que les ministres du culte chrétien appellent la croix. L'empereur voulut donc savoir ce que ce sceau (sèmantron) représentait pour les barbares. Ils déclarèrent l'avoir reçu de leurs mères : une peste féroce s'étant en effet établie chez les Scythes orientaux, il fallut que des chrétiens conseillent de tatouer le front des adolescents avec le signe en question. Les barbares n'ayant nullement dédaigné ce conseil, ils recouvrèrent la santé grâce à lui ». ${ }^{137}$

Cet épisode procède à nouveau d'une interprétation chrétienne : les mercenaires recrutés par les Perses séditieux étaient vraisemblablement pourvus de petits tatouages thérapeutiques cruciformes analogues à ceux que nous avons rencontrés en Irak. Les Byzantins ont tout naturellement attribué cette judicieuse médication à la bienveillance chrétienne.

Ces deux anecdotes donnent une résonance ancienne aux emplois thérapeutiques ou prophylactiques du tatouage constatés par l'ethnographie moderne à côté du tatouage nubile féminin. Elles permettent par analogie d'inférer l'existence de tatouages thérapeutiques à côté du tatouage ornemental dans les contrées danubiennes et balkaniques. Et si,

\footnotetext{
${ }^{133}$ Mauchamp, 1911, p. 142.

${ }^{134}$ Légey, 1926, p. 115.

135 Herber, 1927 , p. 519.

${ }^{136}$ Herber, 1946, p. 324-326.

137 Théophylacte Simocatta (VII ${ }^{\mathrm{e}}$ s.), Histoires, V, 10, 14-15.
} 
comme nous le croyons, les tatouages d'Ötzi doivent être rattachés à ce groupe danubienbalkanique, il n'est pas impossible que les compagnes de notre alpiniste aient-elles aussi été tatouées. Ce tatouage aura été ornemental, comme il le fut chez les femmes thraces, et plus tard chez les Catholiques bosniaques. Les vases grecs sont pour l'instant les seules pièces archéologiques qui illustrent de façon indiscutable cet ancien tatouage féminin balkani$q^{138}{ }^{138}$. Les statuettes féminines du néolithique découvertes au siècle dernier autour du Danube nous semblent devoir être écartées : leurs motifs peints ou incisés se rapportent davantage à l'agencement des différentes parties du vêtement et au décor du tissu ${ }^{139}$ qu'au tatouage ou à la peinture corporelle proprement dits ${ }^{140}$.

\section{La cautérisation et la question du réseau anatomique}

L'hypothèse diagnostique inédite que le docteur Pascale Hégy propose à la fin de cet article $^{141}$ suppose que les tatouages d'Ötzi ont été appliqués loco dolenti. Nous avons vu que ce principe d'action motivait la quasi-totalité des applications thérapeutiques du tatouage. Certes, Ötzi n'avait sans doute pas conscience du mécanisme propre de la sciatique ; la disposition de ses douleurs pouvait toutefois lui faire supposer l'existence d'un canal ou d'un conduit quelconque véhiculant un agent pathogène depuis le dos jusqu'aux pieds, en passant par les jambes ${ }^{142}$. Bien que l'existence d'un concept de réseau anatomique ne puisse être véritablement inférée du traitement mis en œuvre chez Ötzi, la question mérite d'être soulevée : de tous temps les indices empiriques n'ont pas manqué, qui permettaient d'imaginer plusieurs voies de circulation à travers le corps humain : l'ossature ; les orifices d'ingestion et d'évacuation; les voies respiratoires ; le trajet des veines supérieures et des tendons, souvent perceptible à l'œil nu ; la mobilité apparente de certaines sensations nerveuses, etc. À partir de ces données observables par tous, une culture donnée avait la possibilité de formuler trois types d'hypothèses : topographiques (localisation des diverses ramifications de ce réseau), mécaniques (quelle(s) substance(s) circule(nt) dans ce réseau et comment) et thérapeutiques (comment corriger les disfonctionnements de ce réseau).

\footnotetext{
${ }^{138}$ Zimmermann, 1980.

${ }^{139}$ C'est indiscutablement le cas à Vinča, un site qui a livré de très nombreuses statuettes (Serbie, 48003500 av. J.-C.) : cf. Vasić, 1936, pl. 35, Fig. 173 (ceinture ; jupe décorée de motifs crénelés) ; pl. 58, Fig. 271 (jupe longue décorée de damiers) ; pl. 59, Fig. 286 (ceinture et jupe avec mouvement de plis vers l'avant, rangée de triangles sous la ceinture) ; pl. 81, Fig. 379 (jupe avec figuration de plis verticaux assez réalistes) ; pl. 86, Fig. 402 (grande robe dont plusieurs plis ou coutures descendent depuis les épaules jusqu'aux chevilles); pl. 90, Fig. 422 (chemise col en V, pan de tissu enroulé autour des hanches, jupe tombante avec plis verticaux) ; pl. 98, Fig. 464 (chemisier avec plis réalistes dans le dos et autour des seins), etc. Les interprétations symboliques que Marija Gimbutas donne de ces motifs sont une aberration (Gimbutas, 1989).

${ }^{140} \mathrm{La}$ question s'est posée pour les figurines de Cucuteni (Roumanie, c. 5000 av. J.-C.), entièrement recouvertes d'incisions parallèles. Mais il faut sans doute suivre C. Cohen (2003, p. 141) qui pense tout simplement au drapé d'un vêtement. Les motifs géométriques peints sur les figurines d'Ovcarovo (Bulgarie, $5^{\mathrm{e}}$ millénaire av. J.-C., Cohen, 2003, p. 146-147) pourraient éventuellement laisser place au doute.

${ }^{141}$ Infra, texte de P. Hégy dans ce même numéro la plupart des tatouages d'Ötzi sont situés sur le trajet du nerf sciatique).

${ }^{142}$ La croix tatouée à l'intérieur du genou droit pouvait être intégrée à ce réseau, bien qu'elle semble plutôt correspondre à une douleur arthrosique.
} 
Les plus anciennes tentatives connues sont celles de la médecine égyptienne qui dote le corps humain d'une arborescence complexe de conduits (met) dans lesquels circulent entre autres du sang, des sécrétions (nechout), des substances pathogènes vivantes et quasipersonnelles (ââ, setet, oukhedou), des souffles de mort (deheret) et des souffles de vie. Les textes existants ne permettent pas de savoir s'il existait un consensus sur le nombre et la localisation de ces conduits, ni même d'en reconstituer la topographie précise. La première liste du papyrus Ebers (c. 1500 av. J.-C.) compte une cinquantaine de conduits menant aux divers membres et organes du corps ${ }^{143}$. Une autre section du même papyrus ${ }^{144}$ compte douze conduits pour le cœur, vingt conduits répartis par paires vers les divers points de la tête (front, nuque, yeux, sourcils, narines, oreilles), quatre paires de conduits pour les membres supérieurs et inférieurs. Ces conduits servent parfois à établir des diagnostics et des traitements (potions, évacuants, cataplasmes, onguents, etc.). Certaines recettes montrent que les cataplasmes et les onguents pouvaient être appliqués sur les conduits eux-mêmes ${ }^{145}$, sans déroger au principe de traitement local ${ }^{146}$ de type conjuratoire et magique caractéristique de la médecine égyptienne ${ }^{147}$. Aucune trace en revanche de stimulations chirurgicales (saignée ou cautérisation) exercées sur les conduits-met. Nous allons voir que l'Antiquité occidentale a pourtant utilisé la cautérisation pour dérouter ou stopper la circulation des humeurs pathogènes.

\subsection{La cautérisation répulsive}

Le traité hippocratique Des affections internes (§ 18) attribue notre sciatique moderne à une « grande maladie des veines creuses qui, partant de la tête, se dirigent le long du cou par l'épine dorsale vers la malléole externe du pied et l'entre-deux du gros orteil ». Cette maladie commence par provoquer, du côté droit ou gauche, une douleur au niveau des lombaires, descend peu à peu vers le bas, jusqu'à la malléole du pied et l'entre-deux du gros orteil, puis est censée remonter vers la tête où elle occasionne une céphalée insupportable. Le traité prescrit des potions purgatives. Toutefois, si ce traitement échoue - et dans le cas où la maladie affecterait le côté droit - on conseille de : "cautériser quatre escarres au bas de l'omoplate droite, trois à la cavité de la hanche droite, deux à la fesse, deux au milieu de la cuisse, une au-dessus du genou, une au-dessus de la malléole [externe]. Celui qui cautérise ainsi ne laisse progresser la maladie (nosos) ni vers le haut ni vers le bas. [...] si la douleur (odunè) s'est établie quelque part ailleurs, cautériser : il faut cautériser les parties charnues (sarkôdea) avec des cautères métalliques (sidèria), les parties osseuses et tendineuses (osteôdea kai neurôdea) avec des champignons (mukètes) ». ${ }^{148}$

\footnotetext{
${ }^{143}$ P. Ebers, § 854b-854o (Bardinet, 1995 : p. 88-99).

${ }^{144}$ P. Ebers, § 856a-856h (Bardinet, 1995 : p. 363-364).

145 P. Ebers, § 627-696 (Bardinet, 1995; p. 340-348).

${ }^{146}$ Sauf peut-être $\S 856$ e (cataplasme appliqué sur les conduits de la nuque auxquels on attribue une baisse de la vision).

${ }^{147}$ Les différents produits appliqués sur les endroits malades sont censés chasser les substances et les êtres néfastes qui s'y sont installés. Sur les formules exorcistes qui accompagnent souvent ces applications, cf. Bardinet, 1995, p. 48 sq.

${ }^{148}$ Affections internes, $§ 18$ (voir aussi § 51).
} 
On reconnaît là des analogies incontestables avec la stimulation chirurgicale chinoise $^{149}$. En effet, bien que les points de cautérisation soient répartis sur le trajet sciatique, ils ne sont pas tributaires de la localisation exacte des douleurs du patient au moment du traitement (sauf dans la deuxième partie de la prescription) : le praticien obéit plutôt à une topographie préétablie censée lui permettre de contrarier au mieux la circulation de l'agent pathogène dans «la grande veine creuse » ${ }^{150}$. Cette manière d'exercer par la cautérisation une action répulsive sur telle ou telle partie du système vasculaire est utilisée ailleurs par les médecins grecs pour des douleurs spécifiquement articulaires ${ }^{151}$, mais aussi pour des affections respiratoires : pour empêcher la descente du phlegme et de la bile par les huit veines principales partant de la tête ${ }^{152}$, huit cautérisations - qui sont en même temps des phlébotomies - sont appliquées aux points d'ancrage de ces vaisseaux : « deux près des oreilles, deux aux tempes, deux à l'arrière de la tête de part et d'autre de l'occiput, deux au nez près des encoignures des yeux ${ }^{153}$. C'est à une mécanique semblable qu'Hérodote se réfère pour expliquer les cautérisations prophylactiques des nomades libyens :

«Lorsque leurs enfants ont quatre ans, ils leur brûlent les veines du sommet de la tête avec de la laine graisseuse de mouton, et certains mêmes celles des tempes, la raison de cela étant qu'une fois pour toute le flegme qui s'écoule de la tête ne leur cause plus de maux ; et c'est grâce à cela, disent-ils, qu'ils sont en excellente santé ». ${ }^{154}$

On peut sans doute soupçonner Hérodote de réinterpréter la cautérisation libyenne à la lumière des procédés grecs. Nous avons vu toutefois que les Égyptiens, voisins des Libyens, disposaient depuis longtemps d'un concept de conduits vecteurs de substances pathogènes permettant une étiologie assez proche de celle qu'Hérodote attribue aux Libyens. On a d'ailleurs tout lieu de penser que les différentes interventions chirurgicales mises en œuvre contre les écoulements infectieux - qu'elles soient prophylactiques ou thérapeutiques - remontent à une époque reculée, et que celles que l'on trouve dans la médecine grecque sont des adaptations de pratiques protohistoriques ${ }^{155}$.

\subsection{La cautérisation par combustion organique (moxibustion) en Occident}

On aura noté dans le passage cité plus haut des Affections internes une curieuse cautérisation aux champignons dont la description va nous être donnée par Caelius Aurelianus, un médecin du $\mathrm{V}^{\mathrm{e}}$ siècle qui réunit les divers traitements indiqués par ses prédécesseurs pour la sciatique :

${ }^{149}$ Cf. extrait du Maishu de Zhangjiashan (p. 9) et prescriptions de Chunyu Yi ( ${ }^{\circ} 59$ ).

${ }^{150}$ Pour l'histoire du système vasculaire dans la médecine grecque, cf. Duminil, 1983.

${ }^{151}$ Il s'agit à nouveau de contrarier l'engorgement pathogène des articulations en cautérisant les vaisseaux censés véhiculer une humeur ou à une mucosité mauvaise, « soit largement au-dessus des articulations affectées de rhumatisme, soit au plus près » (Rufus d'Éphèse, II ${ }^{\mathrm{e}}$ s. apr. J.-C., ap. Aétios, Livres médicaux, XII, 25).

152 Décrites dans Nature de l'homme, 11.

${ }^{153}$ Deuxième livre des maladies, 12. Pour arrêter les fluxions oculaires, les médecins grecs pratiquaient aussi de profondes incisions au niveau du front et des tempes (Marganne, 1994 : p. 147-172).

${ }^{154}$ Hérodote, Histoires, IV, 187. Celse connaît aussi la « méthode des Africains » qui consiste à cautériser « le vertex jusqu'à l'os de façon qu'il rende une écaille osseuse » (De la médecine, VII, 7, 15).

${ }^{155}$ Margane, 1994, p. 163. 
«Certains appliquent sur les endroits douloureux des champignons ligneux (lignei fungi) qu'ils raccourcissent en bas et en haut. Les ayant enflammés au sommet, ils les laissent se consumer jusqu'à ce qu'ils soient réduits en cendre et tombent d'euxmêmes. De part sa pénétration douce (lenis penetratio), ce type de cautérisation est modéré. D'autres encore trempent un morceau de toile dans l'eau et en placent un sur chaque endroit [à cautériser]. Ils y apposent alors une planchette de tilleul, coupent de la plante saponaire (strouthion) en petits morceaux, et disposent ensuite sur les planchettes de tilleul ces petits morceaux trempés dans l'huile et embrasés par un tison brûlant. Ils répètent l'opération deux ou trois fois à intervalle régulier jusqu'à ce que le bois carbonise et que la peau se couvre de cloques $\gg .{ }^{156}$

Dioscoride ( $\mathrm{I}^{\mathrm{er}}$ s. apr. J.-C.), dans un petit ouvrage rarement cité, donne à son tour un tableau très complet des anciennes techniques de cautérisation :

«Les cautérisations sont profitables pour les sujets atteints de sciatique : après avoir allumé des fientes de chèvre, appliques-en sur la dépression [située] entre les tendons du pouce, en ayant au préalable appliqué de l'huile ou imbibé d'huile une [compresse de] laine, puis fais brûler [le combustible] en [le] renouvelant jusqu'à ce que la sensation [de chaleur] s'en aille par le bras jusqu'à la hanche $e^{157}$; ou, après avoir façonné une pastille d'argile grande comme une pièce de monnaie et épaisse d'un doigt, et après l'avoir appliquée sur le siège de la douleur, appuies-y un cautère rougi au feu jusqu'à ce que la sensation soit rendue difficilement supportable ; ou bien, après avoir tendu la peau à l'endroit douloureux, divise-la avec un stylet rougi au feu sur une longueur de deux doigts. Les Libyens font, avec de la sciure de lotus, du soufre naturel et un purgatif (élatèrion), une pyramide qu'ils appliquent sur le siège de la douleur : ils préparent un support en double épaisseur (charpie, bandelette, ou lamelle d'os) sur lequel ils posent la pyramide allumée. Les Marmarides [en Libye] cautérisent avec des bois d'olivier humidifiés, en recherchant une chaleur humide qui ne provoque pas de brûlure ». ${ }^{158}$

Ces textes montrent que, contrairement à une idée reçue, la cautérisation par combustion organique - donc la moxibustion ${ }^{159}$ - n'a jamais été une exclusivité asiatique ${ }^{160}$. Le médecin vénitien Prospero Alpino, qui séjourna au Caire entre 1581 et 1584, décrit une

\footnotetext{
${ }^{156}$ Caelius Aurelianus, Maladies chroniques, V, 1, 20.

${ }^{157}$ Notice reprise au $\mathrm{II}^{\mathrm{e}}$ siècle par Archigénès (ap. Aétios, Livres médicaux, XII, 1). Cette cautérisation distale suppose une interaction entre le pouce de la main et la hanche. Démétrios d'Apamée (Alexandrie, III ${ }^{\mathrm{e}}$ s. av. J.-C.) l'appliquait sur la main correspondant à la jambe affectée par la sciatique avec un cautère métallique enveloppé (ap. Caelius Aurelianus, Maladies chroniques, V, 1, 21). Pline attribue une recette voisine de celle de Dioscoride aux «Mages »; la cautérisation doit être selon lui pratiquée sur la main opposée au côté affecté (Histoire naturelle, XXVIII, 198-199). Contre la sciatique, Antyllos (II ${ }^{\mathrm{e}} \mathrm{s}$.) cautérisait le gros orteil jusqu'à l'os avec des fientes de chèvre (ap. Aétios, Livres médicaux, XII, 1).

158 Dioscoride, Remèdes simples, I, 235, 1-3. Si cet ouvrage est bien de Dioscoride, il précède son grand œuvre, la Matière médicale.

${ }^{159} \mathrm{Cf} . \mathrm{n}^{\circ} 51$.

${ }^{160}$ Huard et Wong (1958 : p. 39) ont ainsi pensé que la moxibustion observée par Prospero Alpino en Égypte (cf. n. suiv.) avait été importée d'Asie par les Turcs entre le IX ${ }^{\mathrm{e}}$ et le XIII ${ }^{\mathrm{e}}$ siècles.
} 
technique de cautérisation très répandue chez les Égyptiens et les Nomades du désert (pyramide de coton enroulé dans une bandelette de lin) ${ }^{161}$ apparentée à celle qu'Hérodote avait constatée chez les Libyens nomades (oisupè probatôn : laine graisseuse de mouton). Ces cautérisations nord-africaines ont en commun avec les anciennes prescriptions grecques d'être pratiquées à différents endroits de la tête (sinciput, occiput, derrière les oreilles, sur les tempes) pour contrarier les écoulements responsables d'affections respiratoires ou oculaires. Prospero Alpino signale aussi leur application locale pour des douleurs chroniques et un affaiblissement dans les articulations. Les observations postérieures confirment l'importance de la cautérisation (mais aussi des scarifications simples) dans la pratique médicale des populations nord-africaines et proche-orientales ayant conservé leur organisation tribale ${ }^{162}$.

\subsection{La cautérisation en Inde}

L'Inde a elle aussi hérité de la cautérisation, à l'instar du Tibet ${ }^{163}$. On trouve dans le Corpus de Suśruta ${ }^{164}$ une liste réunissant divers objets métalliques, mais aussi des baguettes de bois et d'autres végétaux (poivre long), des fientes de chèvres, des objets solides (dent de vache) ou des liquides (miel, huile, mélasse) préalablement chauffés. La cautérisation intervient à la manière de l'ancienne moxibustion chinoise pour corriger « une manifestation particulièrement agitée de vent vital (váyu) ayant son siège dans la peau, la chair, une veine, les nerfs et les articulations et donnant lieu à d'abominables douleurs internes ». ${ }^{165}$

Une autre indication rappelle de son côté les diverses cautérisations que les Grecs prescrivaient contre les descentes d'humeurs céphaliques :

«Les arcades sourcilières, le front et les os temporaux doivent être cautérisés en cas de maladies affectant la tête, comme en cas d'ophtalmie ». ${ }^{166}$

À la fin du XIX ${ }^{\mathrm{e}}$ siècle, Édgard Thurston observa parmi les tribus du sud de l'Inde une grande variété de procédés de cautérisation ${ }^{167}$. Avec des aiguilles chauffées, des petits cigares végétaux ou des mèches imbibées d'huile, on administre plusieurs pointes de feu à destination prophylactique ou thérapeutique. La femme qui attend son premier enfant en reçoit quatre (au pouce et au poignet de chaque main), le nouveau-né une vingtaine ${ }^{168}$ ou

${ }^{161}$ Prospero Alpino, La médecine des Égyptiens, III, 12, (texte latin, Venise, 1591 : p. 97-98).

$162 \mathrm{Cf}$. entre autres, Strouhal, 1981. La cautérisation organique n'est toutefois plus pratiquée à l'époque moderne $\left(\mathrm{XIX}^{\mathrm{e}}\right.$ et $\mathrm{XX}^{\mathrm{e}} \mathrm{s}$.). Cette évolution doit être mise sur le compte de l'influence exercée successivement par les médecines (et les industries) grecque, arabe puis occidentale.

163 Cf. p. 5.

164 Susrutasamhita, compilation achevée au VII ${ }^{\mathrm{e}}$ siècle après J.-C., mais reprenant des matériaux remontant aux derniers siècles avant notre ère.

165 Susrutasamhita, 12, trad. Bhishagratna, 1907, p. 90.

166 ibid.

167 Thurston, 1906, p. 398-400.

${ }^{168}$ Chez les Mala, après le bain (localisation non-précisée). 
moins ${ }^{169}$. Parfois les pointes de feu sont appliquées à la suite d'une maladie infantile (convulsions ou autres) ${ }^{170}$. Charles-Eugène d'Ujfalvy fit un constat assez similaire chez plusieurs ethnies de la partie pakistanaise du Cachemire (Baltistan, haute vallée de l'Indus) :

«Chez les Baltis et [les] Dardous, on se sert de cautérisations comme de moyens préventifs contre toutes espèces de maux. Ainsi, les mères brûlent aux enfants un petit rond sur le vertex, de la grosseur d'une pièce de 50 centimes. Chez les KafirisSiapochs, ces brûlures se font aussi au-dessus des oreilles. On rencontre beaucoup d'individus avec des marques de brûlures sur le ventre et sur les jambes, cette fois-ci non comme un moyen préventif, mais comme moyen de guérison. [...] Les Tchilâsis, comme les Dardous et les Baltis, ont des petites brûlures sur le sommet de la tête que leurs mères leur font dès la plus tendre enfance, pour prévenir les maladies $\gg .{ }^{171}$

Ces cautérisations céphaliques prophylactiques nous rappellent bien entendu celles qu'Hérodote avait notées chez les enfants libyens. Il s'agit là très vraisemblablement d'une des destinations privilégiées de la cautérisation ancienne.

\subsection{Conclusion}

La cautérisation organique semble donc bien avoir précédé en Europe comme en Asie la cautérisation métallique. Employée dès avant l'époque historique, elle anticipa logiquement la maîtrise de la métallurgie et constitue donc, à l'instar du tatouage (ou des scarifications simples), une des composantes majeures de la petite chirurgie préhistorique. Les Grecs en héritèrent, avant de lui préférer le cautère métallique et de cantonner la cautérisation au rôle hémostatique et antiseptique qu'on lui connaît aujourd'hui. La cautérisation préhistorique avait pourtant des ambitions et des applications plus larges : elle fut sans doute d'abord un contre-irritant local, appliqué pour tout type d'affections sur le siège de la douleur, mais aussi, par raisonnement analogique, un procédé répulsif (brûler le mal). Dès lors que l'on conjectura dans le corps humain une circulation de fluides vitaux ou morbides, les propriétés de la cautérisation purent être mises à profit dans la régulation du réseau anatomique : l'application distale était donc envisageable.

Certaines recettes populaires rassemblées par Pline montrent que l'homo antiquus croyait souvent obtenir un résultat curatif en agissant sur un point éloigné du siège de l'affection ${ }^{172}$. Mais ce genre de thérapeutiques ne repose pas forcément sur un réseau anatomique très élaboré. Le perfectionnement de ce dernier n'a pu réellement s'opérer

\footnotetext{
${ }^{169}$ Aux environs de Mysore et de Salem, l'enfant est cautérisé « près du nombril, sur les pieds, le dos des mains, le visage, la nuque et les flancs ».

${ }^{170}$ Chez les Kathira vandlu et d'autres tribus nomades, on cautérise les enfants victimes des intempéries (affection respiratoire ?) entre les sourcils, sur les tempes et parfois à l'abdomen.

${ }^{170}$ Ujfalvy, 1882, p. 223-224.

${ }^{172}$ Pline, Histoire naturelle, XXVIII, 37 (salive appliquée sous les jarrets contre des douleurs à la nuque), 38 (salive sur la paupière contre l'engourdissement d'un membre), 60 (pour évacuer une poussière dans l'œil, comprimer l'autre œil ; souffler sur le front de quelqu'un qui avalé de travers ; frictionner la nuque contre des douleurs aux jarrets), 61 (entourer de laine les extrémités des membres en cas de saignements de nez; contre les
} 
qu'au sein de médecines bénéficiant des atouts propres de la civilisation : écriture, structuration et homogénéisation des connaissances. L'Égypte, la Grèce et la Chine ont chacune à leur façon développée un réseau anatomique dont le concept-souche était vraisemblablement déjà partagé par d'autres cultures. Toutefois, on ne trouve pas de régulation chirurgicale des conduits-met chez les Égyptiens, alors que leurs voisins Libyens pratiquaient sans doute depuis le néolithique plusieurs sortes de cautérisations organiques. Les Égyptiens - comme du reste les Mésopotamiens - ont surtout privilégié la pharmacologie (onguents, cataplasmes, évacuants). Cela procède peut-être, de la part de ces deux civilisations, d'une désaffection volontaire à l'égard de procédés mutilants comme la cautérisation et le tatouage, restés l'apanage des tribus sans écriture. Cette remarque vaut aussi pour la Grèce : bien que les prescriptions citées plus haut témoignent d'une utilisation délibérée de la cautérisation répulsive, celle-ci n'a jamais été élevée au rang de thérapeutique générale par les disciples d'Hippocrate. Les Scythes frontaliers mentionnés dans Airs, eaux, lieux en faisaient pourtant un large usage ${ }^{173}$, mais leurs nombreuses cicatrices n'étaient certainement pas du goût des Grecs, comme du reste les tatouages barbares mentionnés par Hérodote et d'autres auteurs anciens. À l'époque d'Hippocrate, la phlébotomie elle non plus ne fut pas, comme on l'a souvent cru, un remède primordial et universel $^{174}$. Il faut attendre Galien pour qu'elle prenne l'essor qu' on lui connaît en tant que régulateur des pléthores humorales et sanguines. C'est finalement en Chine, nous l'avons vu, que le concept de réseau anatomique a atteint son plus haut degré de systématisation, et c'est là qu'ont été conservés deux procédés de petite chirurgie eux aussi sans doute répulsifs à l'origine : la puncture et la moxibustion.

\section{Dernières questions}

\subsection{Les champignons transportés par Ötzi}

Toutes les données rassemblées ci-dessus concourent à donner de la cautérisation préhistorique une image assez riche, tant sur le plan des techniques que des prescriptions. La moxibustion, nous l'avons dit, fut évidemment antérieure à la maîtrise de la métallurgie. Toutes sortes de procédés organiques s'offraient à nos ancêtres : les listes conservées par les médecins grecs et indiens nous en ont donné une idée. L'une de ces techniques - la combustion de champignons - a déjà éveillé notre attention et mérite d'être maintenant confrontée au cas particulier d'Ötzi.

Les deux variétés de champignon découvertes aux côtés d'Ötzi ont déjà donné lieu à de nombreuses discussions ${ }^{175}$. La masse de Polypore amadouvier (Fomes fomentarius) ${ }^{176}$ qui occupait la plus grande partie du sac-ceinture en cuir n'a pas semblé poser de problème : les

maux d'estomacs, serrer les pieds ou tremper les mains dans l'eau chaude), 64 (se frotter derrière les oreilles en cas d'ophtalmie, frotter le front en cas de larmoiement).

173 Supra, p. 7.

${ }^{171}$ Cf. l'importante mise au point de Brain, 1986, p. 112-120.

175 Bibliographie exhaustive chez Peinter et al., 1998.

${ }^{176}$ Parasite spécifique des feuillus (hêtres en part., mais aussi frênes, ormes, noyers et bouleaux). 
données ethnographiques et celles de la littérature ancienne ${ }^{177}$ ont permis de lui rendre son rôle traditionnel d'amadou. Les deux morceaux de Piptoporus betulinus (Polypore spécifique du bouleau) enfilés sur des lacets de cuir ont soulevé davantage de questions. On a cru dans un premier temps que ces champignons séchés avaient pu être eux aussi employés comme amorce inflammable. Mais aucune information n'a pu être trouvée sur un tel usage. De fait, les propriétés inflammables du P. betulinus, bien moindres que celles du $F$. fomentarius, ne sont pas supérieures à celles du papier ${ }^{178}$. L. Capasso prétendit en 1998 que le $P$. betulinus possédait des substances actives purgatives et antibactériennes : Ötzi aurait ingéré ce champignon pour combattre la vermine (Trichuris trichiura) qui colonisait ses intestins ${ }^{179}$. Des botanistes suédois (Håkan Tunón, Ingvar Svanberg) et autrichiens (Reinhold Pöder, Ursula Peintner) ont apporté un ferme démenti aux conjectures de L. Capasso $^{180}$ : d'une part les substances et les propriétés chimiques que ce dernier croyait pouvoir trouver dans le $P$. betulinus sont inexistantes, d'autre part les parasites intestinaux d'Ötzi ne provoquent aucun dérangement majeur nécessitant la mise en œuvre d'un traitement spécifique.

L'équipe autrichienne en charge d'analyser les champignons trouvés au Similaun a réuni toutes les données ethnomycologiques et pharmacologiques disponibles relatives au $P$. betulinus $^{181}$. Avec d'autres variétés, le Polypore du bouleau était préparé et consommé par les habitants du Kamtchatka au XVIII ${ }^{\mathrm{e}}$ siècle. Au Sud de Londres (Surrey), découpé en lamelles, il servait de coussinet pour les cors aux pieds ou d'astringent. On prêtait également à son charbon des vertus antiseptiques. Des exemplaires desséchés de $P$. betulinus étaient aussi utilisés pour aiguiser les rasoirs. Enfin, à partir de la seconde moitié du $\mathrm{XX}^{\mathrm{e}}$ siècle, des tests menés en laboratoire ont montré que plusieurs acides extraits de ce champignon exerçaient sur les animaux une action antimicrobienne et anti-inflammatoire.

U. Peintner et al. ne mentionnent pas d'emplois du Polypore de bouleau comme combustible pour la cautérisation. Ils connaissent, par l'intermédiaire de Reginald Buller ${ }^{182}$, la cautérisation aux champignons de la Collection hippocratique, et, comme ce dernier, la classent parmi les emplois du Polypore amadouvier (F. fomentarius). Le traité des affections internes ne précise pourtant pas la variété utilisée ${ }^{183}$ et Caelius Aurelianus parle seulement de champignons poussant sur les arbres (lignei fungi $)^{184}$. Nous avons cru un moment que le $P$. betulinus avait pu être utilisé pour la cautérisation, en nous fondant sur deux observations anciennes faites en Sibérie. Mais les désignations d'amadou de bouleau et de champignon de bouleau que l'on trouve dans ces notices ne doivent rien au $P$. betulinus : elles désignent bien le $F$. fomentarius dont l'hôte principal est, dans ces régions, le bouleau ${ }^{185}$. La première de ces notices provient du jeune Stepan Petrovitch Kracheninnikov qui participa au début des années 1740 à l'exploration du Kamtchatka aux côtés de Georg Wilhelm Steller. Il rapporte des Kamtchadales :

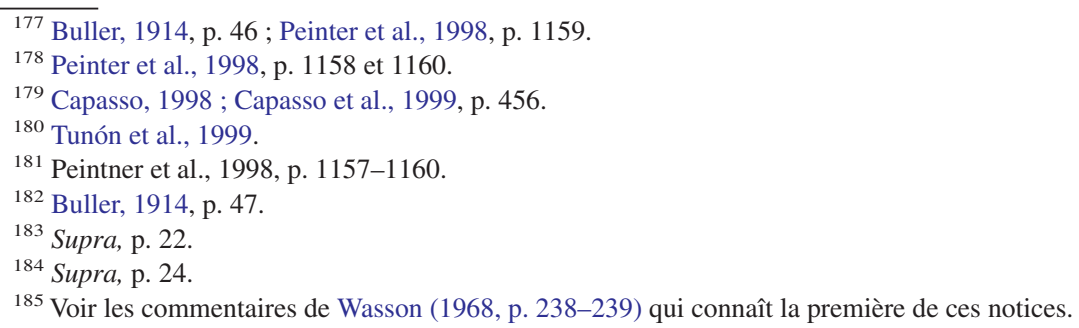


«Contre le rhumatisme, (от лому), ils font des cautérisations sur les endroits affectés avec de l'amadou de bouleaи (из березового труду). Quand cet amadou se consume jusqu'à la chair, il saute avec une grande vivacité ; la chair à cause de cela s'ulcère et il se produit un profond escarre sur lequel certains saupoudrent de la cendre d'amadou (трудовой пепел), alors que d'autres ne font aucun soin. Cette médication (лекарство) est connue dans toute la Sibérie ». ${ }^{186}$

La seconde notice concerne les Ougriens de l'Ob, d'après le rapport de Peter Simon Pallas. Les Ostiaks, nous l'avons vu, pratiquaient le tatouage thérapeutique, mais aussi la moxibustion aux champignons :

«Le principal traitement qu'ils emploient habituellement pour les membres douloureux, enflés et inflammés - affections fréquentes chez eux - c'est d'effectuer des scarifications (Schröpfen), ou de faire brûler sur les endroits souffrants un morceau d'amadouvier (Birkenschwamm) - appelé Jachani - à la manière des moxas orientaux (nach Art der orientalischen Moxa). Mais puisque avec ce traitement l'enflure qui en résulte risque de se produire juste à l'endroit où siège le mal, ils prennent d'abord un charbon ardent et l'approchent de différents endroits de la peau à proximité de la douleur jusqu'à ce qu'ils trouvent un endroit où le malade ne sente pas tant la brûlure; puis ils placent là le cautérisant adéquat qui doit agir et être courageusement supporté par le malade jusqu'à ce que la peau ait été entièrement carbonisée et ait éclaté ». ${ }^{187}$

Mais revenons au problème des deux fragments de $P$. betulinus. C'est auprès d'un botaniste américain qu'Ursula Peintner et ses collègues ont finalement trouvé les parallèles ethnomycologiques les plus intéressants. R.A. Blanchette a récemment inventorié une cinquantaine d'objets collectés au XIX ${ }^{\mathrm{e}}$ siècle chez les Indiens d'Amérique du Nord et décorés avec un polypore du saule (Haploporus odorus, à la senteur anisée caractéristique ${ }^{188}$. Séché, ce polypore était découpé en morceaux de forme ronde ou ovale. Ces portions étaient enfilées sur des lacets de cuir ou des cordelettes puis attachées aux tuniques sacrées, aux colliers de scalps humains ou aux bourses médicinales ${ }^{189}$. Ce champignon était utilisé jusqu' au $\mathrm{XX}^{\mathrm{e}}$ siècle par les guérisseurs indiens (infusions, fumigations, usage hémostatique), et porté parfois comme collier prophylactique.

Les objets décrits par R.A. Blanchette offrent des analogies indéniables avec les deux fragments perforés retrouvés auprès d'Ötzi. Notons que les attaches en cuir de ces derniers sont assez perfectionnées : le fragment $a$ (de forme plus ou moins conique) est traversé par deux lanières ${ }^{190}$ dont l'une passe à travers les cinq perforations de l'autre, et toutes deux forment un nœud complexe qui retient le fragment à son extrémité sommitale. La lanière du fragment $b$ (de forme globulaire) se termine d'un côté par un nœud simple, de l'autre par

\footnotetext{
${ }^{186}$ Kracheninnikov, 1755 (1949), ch. 18, p. 443.

${ }^{187}$ Pallas, 1776, p. 50-51.

${ }^{188}$ Blanchette, 1997 (une cinquantaine d'objet répartis dans trois musées canadiens et un musée américain).

${ }^{189}$ Ces bourses sont des emballages d'objets associés à des rituels et censés posséder des pouvoirs spirituels ou prophylactiques.

${ }^{190}$ Des touffes de poils subsistent près des perforations : il s'agissait donc à l'origine de lanières de fourrure.
} 
une fente de 1,5 cm de long dans laquelle est inséré un élément de cuir composé de trois lanières plus courtes. Les morceaux de $P$. betulinus, retenus par des nœuds, semblent donc avoir été attachés de manière assez définitive. Il aurait été plus simple, dans le cas où l'on souhaiterait seulement en transporter quelques-uns et les destiner à la consommation ou à la combustion, soit de les enfiler sur une courroie simple, soit même de les remiser tels quels dans une besace, sans perforation. Les attaches élaborées sur lesquelles les morceaux de P. betulinus ont été retrouvés militent par conséquent plus en faveur d'une destination culturelle (décoration, rôle symbolique ou magique) qu'en tant qu'objet consommable (nourriture, pharmacologie, combustible).

S'agissant du F. fomentarius, le consensus actuel nous semble tout à fait recevable : cet amadou aura été utilisé comme tel. Ötzi transportait d'ailleurs un morceau de pyrite ${ }^{191}$ qui n'a pas été retrouvé, mais dont la présence à côté du $F$. fomentarius a été déduite des microparticules cristallines observées à la surface du champignon ${ }^{192}$. Cette hypothèse solide ne contredit pas celle, plus fragile, que nous aimerions proposer pour finir. Les données rassemblées dans cette étude montrent que la moxibustion à l'amadou a toutes les chances d'être préhistorique. Attestée chez les médecins grecs, nous venons de la retrouver au XVIII ${ }^{\mathrm{e}}$ siècle en Sibérie ${ }^{193}$. Un pasteur irlandais du XVII ${ }^{\mathrm{e}}$ siècle l'a également notée chez les Indiens de Virgine ${ }^{194}$. Enfin, Ilmari Manninen a réunis un grand nombre d'observations montrant que la moxibustion à l'amadou était fréquemment utilisée par les Lapons, et même par certains Russes (depuis le $\mathrm{XV}^{\mathrm{e}}$ siècle au moins). Ces derniers utilisaient parfois des feuilles d'armoise, comme les Turco-mongols de Sibérie méridionale et d'Asie centrale ${ }^{195}$. Chez les Lapons, la moxibustion à l'amadou, qui laisse des cicatrices, est essentiellement utilisée contre les affections rhumatismales et les douleurs dorsales. Appliquée sur les tempes ou la mâchoire, elle est également censée soigner la céphalée et le mal de dents. À noter que les Lapons cautérisaient aussi la dépression située entre le pouce et l'index, comme certains médecins grecs ${ }^{196}$.

On peut se demander si Ötzi n'aura pas lui-même reçu des moxas de $F$. fomentarius en traitement complémentaire de sa sciatique, comme le feront après lui plusieurs médecins grecs. Ces derniers réservaient la cautérisation à l'amadou pour les parties délicates où affleurent os et tendons, car ils l'estimaient plus modérée que la métallique, et donc sans doute moins mutilante. Mais le témoignage des Grecs sur les cicatrices des Scythes et la description des profondes escarres sibériennes donnée par S.P. Kracheninnikov et S. Pallas peuvent nous faire douter que le derme d'Ötzi ne soit jamais sorti indemne d'un tel

${ }^{191}$ Sulfure naturel de fer que l'on frappe avec un silex pour produire des étincelles.

192 Spindler, 1993 (1994), p. 110.

${ }^{193}$ L'amadou ferait encore partie des combustibles utilisé par les Chinois et les Japonais pour la moxibustion (Peintner et al., 1998 ; p. 1159).

${ }^{194}$ Lettre de John Clayton datée de 1687 et publiée dans les Philosophical Transactions of the Royal society of London, 41, 1, 1744, p. 149 : "As the East-Indians use Moxa, so these burn with Punk, which is the inward Part of the Excrescence or Exuberance of an Oak".

${ }^{195}$ Manninen, 1933. L'auteur, qui ignore les sources anciennes sur les Scythes et les propres recettes des Grecs, est conduit à localiser le foyer de diffusion de la moxibustion en Asie.

${ }^{196}$ Cf. supra, note 157. Chez les Lapons, cette intervention est censée agir contre le mal de dents. Il s'agit donc, dans les deux cas, d'une cautérisation distale. 
traitement ${ }^{197}$. À moins qu'Ötzi ait protégé sa peau avec un matériau isolant, comme le faisaient les Libyens mentionnés par Dioscoride ${ }^{198}$. De fait, encore de nos jours, les médecines tibétaine et chinoise emploient des précautions analogues qui empêchent que la moxibustion laisse des cicatrices ${ }^{199}$.

\section{2. Ötzi et les marques corporelles}

S'il est vrai qu'Ötzi souffrait de sciatique et qu'il a été tatoué loco dolenti, comme le suggère l'hypothèse diagnostique du Dr Hégy, alors les meilleurs parallèles ethnographiques sont à rechercher auprès des ethnies traditionnelles éparpillées autour du bassin méditerranéen (Maghreb, Égypte, Irak) : chez ces dernières, on tatoue les zones douloureuses avec des motifs tout aussi élémentaires que ceux d'Ötzi (ou même identiques : traits isolés ou parallèles, croix), et ce tatouage thérapeutique ou prophylactique - dont les sources byzantines ont montré l'ancienneté - coexiste depuis l'Antiquité avec le tatouage ornemental féminin. Semblables pratiques ont également dû prévaloir au néolithique sur les rives septentrionales de la Méditerranée : le tatouage féminin est abondamment documenté en Thrace, et les auteurs anciens signalent sa présence jusque sur les contreforts occidentaux des Alpes dinariques. En rattachant Ötzi à cette aire danubienne et balkanique, on peut par conséquent imaginer qu'à son époque le tatouage décoratif était déjà réservé aux femmes, tandis que le tatouage thérapeutique s'appliquait indifféremment aux deux sexes.

D'autres mutilations tégumentaires comme la scarification, la puncture ou la perforation - dont le tatouage n'est finalement qu'une variante (ajout d'un colorant) - ont peut-être également été employées en Occident dès l'époque protohistorique, bien que les données relatives à la puncture et à la perforation soient essentiellement asiatiques (Arctique, Sibérie, Chine) et ne nous permettent pas de remonter plus haut que le II $^{\mathrm{e}}$ siècle avant notre ère. Quoi qu'il en soit, la topographie des tatouages d'Ötzi n'a pas lieu d'être comparée à celle des points d'acumoxa, et cela pour deux raisons. La première est d'ordre statistique : les 670 points répertoriés par la médecine chinoise et la cinquantaine de traits tatoués sur Ötzi ont une probabilité de coïncidence qui n'est pas significative ; la seconde est d'ordre historique : le canon topographique des points d'acumoxa n'est pas antérieur au début de notre ère.

Sans doute les compatriotes d'Ötzi connaissaient-ils déjà la cautérisation organique à l'amadou (moxibustion de champignons) attestée chez les anciens Grecs, les Scythes, et chez plusieurs peuples d'Europe du Nord et de Sibérie. L'ancienne cautérisation thérapeutique, souvent administrée loco dolenti, pouvait aussi être distale et prophylactique (Libye,

${ }^{197}$ Nous ne savons pas que l'on se soit soucié d'établir un inventaire des diverses meurtrissures - post mortem ou non - repérables sur l'épiderme d'Ötzi, à l'exception des tatouages.

198 Supra, p. 24 (charpie, bandelette, ou lamelle d'os). Dans cette même notice, Dioscoride dit que les Marmarides de Libye obtiennent, avec du bois d'olivier humidifié, une chaleur qui ne brûle pas.

${ }^{199}$ On brûle un cône pyramidal de moxa sur du sel, une tranche de gingembre ou d'ail, sur des pâtes à base de poivre, de moutarde, de graines d'aconit ou de haricot. Ou bien, sans isolant, on enlève le moxa dès que le patient sent une forte chaleur, et on le renouvelle sans rechercher la brûlure, mais seulement un rougissement de la peau. La moxibustion profonde et mutilante est rarement utilisée : on laisse dans ce cas le moxa se consumer jusqu'à la peau, et on renouvelle plusieurs fois l'opération (Penka, 1987 : p. 23-25). 
Inde). Dans ce cas, peut-on déjà parler d'un concept de réseau anatomique ? Les premiers linéaments de ce concept n'étaient pas difficiles à esquisser, même à l'époque d'Ötzi : il suffisait de se poser la question élémentaire de la pénétration et de la circulation des substances pathogènes dans le corps humain. À cette question, les interventions de petite chirurgie étaient susceptibles d'offrir des réponses thérapeutiques de type analogique (percer, brûler le mal) ou mécanique (ouvrir pour évacuer le mal). Et à partir du moment où l'on imagina une circulation de substances pathogènes, ces interventions répulsives - ou conjuratoires (Pierre Thillaud) - purent être opérées à plus ou moins grande distance du siège de la douleur symptomatique, et prendre parfois la forme d'interventions prophylactiques. La systématisation et le perfectionnement du concept de réseau anatomique semblent toutefois réservés aux cultures qui ont eu la possibilité d'accumuler et d'archiver leurs connaissances par écrit. Or celles-ci, à l'exception notable de la Chine, n'ont pas donné la précellence aux interventions chirurgicales : l’Égypte, la Mésopotamie et la Grèce ont privilégié la pharmacologie et semblent avoir très tôt respecté un tabou propre aux civilisations occidentales : la mutilation corporelle représente pour elles le stigmate d'une humanité dévaluée, celle des Barbares, des captifs et des esclaves. Ce scrupule n'est pas étranger à la Chine : au XIII ${ }^{\mathrm{e}}$ siècle, l'historien chinois Ma Tuan Lin compile des documents anciens $\left(\mathrm{VI}^{\mathrm{e}}-\mathrm{VII}{ }^{\mathrm{e}} \mathrm{s}\right.$.) où les tatouages des Japonais du Nord-Est sont comparés à des zébrures animales ${ }^{200}$. Et de fait, la stimulation chirurgicale chinoise n'est pas mutilante : l'acupuncture ne laisse pas de traces, et la moxibustion produit très rarement des escarres. Le tabou de la marque corporelle constitue donc selon nous la meilleure explication au fait que le tatouage n'ait pas survécu parmi les procédés thérapeutiques des grandes civilisations.

\section{Remerciements}

Cet article a bénéficié du soutien et des conseils du Dr Pierre-Léon Thillaud, chargé de conférences en Paléopathologie à l'École Pratique des Hautes Études ( $4^{\mathrm{e}}$ section). Nous tenons aussi à remercier Fernand Meyer, directeur d'étude à l'École Pratique des Hautes Études ( $4^{\mathrm{e}}$ Section), Sciences et Civilisation du monde tibétain, pour ses informations relatives à la médecine tibétaine.

\section{Références}

Der Mann im Eis, vol. 1, 1992 : Bericht über das Internationale Symposium 1992 in Innsbruck, éd. F. Höpfel W. Platzer, K. Spindler (Veröffentlichungen der Universität Innsbruck, 187), Innsbruck (Autriche), 1992.

Der Mann im Eis, vol. 2, 1995 : Neue Funde und Ergebnisse, éd. K. Spindler, E. Rastbichler-Zissernig, H. Wilfing, D. zur Nedden, H. Nothdurfter Veröffentlichungen des Forschungsinstituts für Alpine Vorzeit der Universität Innsbruck, 2), Vienne (Autriche) / New York, 1995.

\footnotetext{
${ }^{200}$ Hervey de Saint-Denys, 1876, p. 60 et p. 404-405. Cet ouvrage mentionne d'autres peuples méridionaux tatoués.
} 
The Man in the Ice, vol. 3, 1996 : Human Mumies. A global Survey of their Status and the Techniques of Conservation, éd. K. Spindler, H. Wilfing, E. Rastbichler-Zissernig, D. zur Nedden, Hans Nothdurfter (Veröffentlichungen des Forschungsinstituts für Alpine Vorzeit der Universität Innsbruck, 3), Vienne (Autriche) / New York.

Arnaud, B., 2002. Contre-enquête sur la mort d'Ötzi. Sciences et Avenir 661, 96-99.

Attia, A., Buisson, G., 2003. Édition de texte : « Si le crâne d'un homme contient de la chaleur, deuxième tablette ». Journal des médecines cunéiformes 1, 1-24.

Bardinet, T., 1995. Les papyrus médicaux de l'Égypte pharaonique. Traduction intégrale et commentaire, Paris, Fayard.

Batut, L., 1893. Du tatouage exotique et du tatouage en Europe. Archives d'anthropologie criminelle, de criminologie et de psychologie normale et pathologique 8, 77-92.

Bhishagratna, K.K.L., 1907. An English Translation of the Sushruta Samhita Based on Original Sanskrit Text, 1. Sutrasthanam, Calcutta (Inde).

Blanchette, R.A., 1997. Haploporus Odorus: a sacred Fungus in traditional native american culture of the Northern Plains. Mycologia (New York) 89, 233-240.

Brain, P., 1986. Galen on Bloodletting. A Study of the Origins, Development and Validity of his Opinions, with a Translation of the Three Works, Cambridge, Londres, New York. Cambridge University Press.

Buller, A.H.R., 1914. The Fungus Lore of the Greeks and Romans. Transactions of the British Mycological Society 5, 21-66.

Capasso, L., Capelli, A., Frati, L., Mariani-Costantini, R., 1992. Notes on the Paleopathology of the Mummy from Hauslabjoch, Val Senales (Southern Tyrol, Italy). Der Mann im Eis 1, 209-213.

Capasso, L., 1993. A Preliminary Report on the Tattoos of the Val Senales Mummy (Tyrol, Neolithic). Journal of Paleopathology 5, 173-182.

Capasso, L., 1998. 5300 Years Ago, the Ice Man Used Natural Laxatives and Antibiotics. The Lancet 352, 1864.

Capasso, L., La Verghetta, M., D’Anastasio, R., 1999. L'homme du Similaun : une synthèse anthropologique et paléthnologique. L'Anthropologie 103, 447-470.

Chabelard, R., 1940. Le tatouage des indigènes en Afrique du Nord. Université d'Alger, Faculté mixte de Médecine et de Pharmacie, Alger (Algérie) Thèse de doctorat.

Cohen, C., 2003. La femme des origines. Images de la femme dans la préhistoire occidentale. Belin-Herscher, Paris.

Czapkiewicz, M., 1962. Tätowierung bei den irakischen Arabern. Folia orientalia (Cracovie) 4, 41-46.

Dorfer, L., Moser, M., Spindler, K., Bahr, F., Egarter-Vigl, E., Dohr, G., 1998. 5200-Year-Old Acupuncture in Central Europe? Science 282, 242-243.

Dorfer, L., Moser, M., Bahr, F., Spindler, K., Egarter-Vigl, E., Giullén, S., Dohr, G., Kenner, T., 1999. A Medical Report from the Stone Age? The Lancet 354, 1023-1025.

Duminil, M.-P., 1983. Le sang, les vaisseaux, le cœur dans la collection hippocratique. Anatomie et physiologie. Belles Lettres, Paris.

Elper, D.C., 1980. Bloodletting in Early Chinese Medicine and Its Relation to the Origin of Acupuncture. Bulletin of the History of Medicine 54, 337-367.

Field, H., 1958. Body-Marking in Southwestern Asia. Papers of the Peaboy Museum of Archaeology and Ethnology, 45. Harvard University, Cambridge, pp. 1.

Fortuine, R., 1985. Lancets of Stone: Traditional Methods of Surgery among the Alaska Natives. Arctic Anthropology 22, 23-45.

Fouquet, D., 1898. Le tatouage médical en Égypte. Archives d'Anthropologie criminelle, de Criminologie et de Psychologie normale et pathologique 13, 270-279.

Gimbutas, M., 1989. The Language of the Goddess. Harper and Row, San Francisco.

Gmelin, J.G., 1751-1752. Reise durch Sibirien von dem Jahr 1733 bis 1743. A. Vandenhoecks, Göttingen (Allemagne).

Griffiths, W.G., 1946. The Kol Tribe of Central India. The Royal Asiatic Society of Bengal Monograph Series 2, Calcutta (Inde).

Gupte, B.A., Rose, H.A., 1902. Notes on Female Tattoo Designs in India. The Indian Antiquary 31, $293-298$.

Harper, D.J., 1998. Early Chinese Medical Literature. The Mawangdui Medical Manuscripts. P. Kegan, Londres, New York. 
He, Z., Lo, V., 1996. The Channels: a Preliminary Examination of a Lacquered Figurine from the Western Han Period. Early China 21, 81-124.

Herber, J., 1927. Origine et signification des tatouages marocains. L'Anthropologie 37, 517-525.

Herber, J., 1946. Les tatouages de la face chez la Marocaine. Hesperis 33, 323-351.

Herrero, P., 1984. La thérapeutique mésopotamienne. Édition posthume par M. Sigrist Recherche sur les civilisations, mémoire 48, Paris..

Hervey de Saint-Denys, L., 1876. Ma Tuan Lin, Ethnographie des peuples étrangers à la Chine, 1, Genève, H. Georg, Paris, E. Leroux, Londres, Trübner and Co (trad.).

Hoizey, D., 1988. Histoire de la médecine chinoise, des origines à nos jours. Payot, Paris.

Huard, P., Wong, M., 1958. Cautérisation ignée et moxas en Chine, au Japon, dans le proche et moyen-Orient, en Europe. Histoire de la médecine 8, 7-41.

Kannisto, A., 1933. Über die Tatuierung bei den Ob-ugrischen Völkern. Suomalais-ugrilaisen Seuran Toimituksia. Mémoires de la Société finno-ougrienne (Helsinki) 67, 159-185.

Kracheninnikov, S.P., 1755. (Описание земли Камчатки) Description de la terre de Kamtchatka. (rééd. Académie soviétique des Sciences, Moscou), Saint-Pétersbourg (1949).

Krutak, L., 1999. St. Lawrence Island joint-tattooing: Spiritual. Medicinal functions and Intercontinental possibilities. Études inuit, Inuit Studies 23, 229-252.

Kunzang, J., 1973. Tibetan Medicine illustrated in original texts. University of California Press, Berkeley, Los Angeles.

Lantis, M., 1984. Aleut. In: Damas, D. (Ed.), Arctic. Handbook of North American Indians, 5, pp. 161-184 Washington.

Légey, F., 1926. Essai de folklore marocain. Geuthner, Paris.

Lo, V., 2001. The influence of nurturing life culture on the development of Western Han acumoxa therapy. In: Hsu, E. (Ed.), Innovation in Chinese Medicine, 3. Needham Research Institute Studies, Cambridge, pp. 19-50.

Lo, V., 2002. Spirit of Stone: Technical Considerations in the Treatment of the Jade Body. Bulletin of the School of Oriental en African Studies 65, 99-128.

Luard, C.E., 1904. Tattooing in Central India. The Indian Antiquary 33, 219-221 (sept.); 237-249 (oct.); 301-309 (déc.).

Manninen, I., 1933. Über die Moxibustion in der Volksmedizin der Nordvölker. Suomalais-ugrilaisen Seuran Toimituksia. Mémoires de la Société finno-ougrienne (Helsinki) 67, 256-266.

Marsh, G.H., Laughin, S.L., 1956. Human Anatomical Knowledge among the Aleutian Islanders. Southwestern Journal of Anthropology 12, 38-78.

Marinis de, R.C., 1997. Les statues-stèles et les statues-menhirs de la région alpine à l'âge du Cuivre. Dossiers d'Archéologie 224, 52-63.

Marganne, M.-H., 1994. L’ophtalmologie dans l'Égypte grécoromaine d'après les papyus littéraires grecs. Brill, Leyde, New York, Cologne.

Mauchamp, É, 1911. La sorcellerie au Maroc. CEuvre posthume précédée d'une étude documentaire sur l'auteur et l'œuvre par Jules Bois. Dorbon-Ainé, Paris.

Meyer, F., 1988. Gso-ba rig-pa. Le système médical tibétain. Presses du CNRS, Paris.

Pallas, P.S., 1776. Reise durch verschiedene Provinzen des rußischen Reichs. Académie impériale des Sciences, Saint-Pétersbourg $3^{\mathrm{e}}$ partie (années 1772-1773).

Peinter, U., Pöder, R., Pümpel, T., 1998. The Iceman's Fungi. Mycological Research 102, 1153-1162.

Penka, C., 1987. La moxibustion. G. Trédaniel, Paris.

Polosmak, N.V., 2000. Tattoos in the Pazyryk World. Archaeology, Ethnology and Anthropology of Eurasia (Novosibirsk) 4, 95-102.

Quilici, B., 2002. Ötzi, l'homme des glaces (documentaire francobritannique de 50 minutes, diffusé sur France 5 le 25/10/2002) (production et réalisation)..

Rolle, R., 1992. Die Skythenzeitlichen Mumienfunde von Pazyryk - Forst-Konservierte Gräber aus dem Altaigebirge. Der Mann im Eis, 1, pp. 334-358.

Rudenko, S.I., 1949a. (Татуировка азиатских Эскимосов) Le tatouage des Esquimaux asiatiques. (Советская Этнография) Ethnographie soviétique 1, 149-154.

Rudenko, S.I., 1949b. (Древнейшая “скифская” татуировка) Les anciens tatouages "scythes”. (Советская Этнография) Ethnographie soviétique 3, 133-143. 
Rudenko, S.I., 1970. Frozen Tombs of Siberia: the Pazyryk Burials of Iron Age Horsemen (trad. du russe M.W. Thompson, avec révisions de l'auteur). J.M. Dent and Sons, Londres.

Sjøvold, T., 1992. Einige statistische Fragestellungen bei der Untersuchung des Mannes vom Hauslabjoch. Der Mann im Eis, 1, pp. 188-197.

Sjøvold, T., Bernhard, W., Gaber, O., Künzel, K.-H., Platzer, W., Unterdorfer, H., 1995. Verteilung und Größe der Tätowierungen am Eismann von Hauslabjoch. Der Mann im Eis, 2, pp. 279-286.

Smeaton, W., 1937. Tattooing among the Arabs of Iraq. American Anthropologist 39, 53-61.

Spindler, K., 1993. Der Mann im Eis: die Ötztaler Mumie verrat die Geheimnisse der Steinzeit, Munich, Allemagne (trad. E. Osers, 1994. The Man in the Ice. The Preserved Body of a Neolithic Man Reveals the Secrets of the Stone Age, Londres, Weidenfeld and Nicolson) (1994)..

Spindler, K., 1997. L'homme gelé, une momie de 5000 ans dans un glacier des Alpes de l'Ötztal. Dossiers d'Archéologie 224, 8-27.

Steller, G.W., 1774. Beschreibung von dem Lande Kamtschatka. Leipzig, J. G. Fleischer, Francfort.

Stone, R., 2000. Ice Man Warms Up for European Scientists. Science 289, 2254.

Strouhal, E., 1981. Folk Medicinal Treatment in Egyptian Nubians. Annals of the Náprstek Museum (Prague) 10, $183-193$.

Thurston, E., 1906. Ethnographic Notes in Southern India, Madras (Inde). Asian Educational Services.

Truhelka, C., 1896. Die Tätowirung bei den Katholiken Bosniens und der Hercegovina. Wissenschaftliche Mittheilungen aus Bosnien und der Hercegovina 4, 493-508.

Tunón, H., Svanberg, I., Pöder, R., Peintner, U., 1999. Laxatives and the Ice Man. The Lancet 353, 925-926.

Ujfalvy, C.-E., 1882. Voyage dans l'Himalaya occidental (le Koulou, le Cachemire et le petit Thibet). Bulletin de la Société d'Anthropologie de Paris 5, 217-226.

Unschuld, P.U., 1985. Medicine in China. A History of Ideas. Comparative studies of health systems and medical care, 13. Berkeley. University of California Press, Los Angeles, Londres.

Unschuld, P.U., Latarjet, B., Schuster, P.-K., Desroches, J.-P., Obringer, F., 2001. Médecines chinoises. Indigène éditions, Paris.

Van der Velden, E., Den Dulk, L., Leenders, H., Dingemans, K., Van der Bergh Weerman, M., Van der Putte, S., Vuzevski, V., Naafs, V., 1995. The Decorated Body of the Man from Hauslabjoch. Der Mann im Eis, 2, pp. 275-278.

Vasić, M.M., 1936. (Преисториска Винча) t. 3: (Пластика - теракоте) - [Vinča préhistorique, 3 : Plastique - Modelages -], Belgrade..

Veniaminov, I.E., 1840. (Записки объ островахъ Уналашкинскаго отдела) Saint-Pétersbourg, trad. L.T. Black et R.H. Geoghegan, 1984. Notes on the Islands of the Unalashka District. Alaska History, 27 Kingston (Ontario, Canada).

Wasson, R.G., 1968. Soma: Divine Mushroom of Immortality. H.B. Jovanovitch, New York Ethno-mycological Studies, 1.

Zhang, R., Wu, X., 1992. Les points d'acupuncture et leur mécanisme d'action. Masson, Paris Institut de médecine traditionnelle chinoise de Pékin, trad. F. Marmori.

Zimmermann, K., 1980. Tätowierte Thrakerinnen auf griechischen Vasenbildern. Jahrbuch des deutschen archäologischen Instituts 95, 163-196.

Zur Nedden, D., Wicke, K., 1992. Der Eismann aus der Sicht der radiologischen und computertomographischen Daten. Der Mann im Eis, 1, pp. 131-148. 